Article

\title{
Chemical Composition and Antimicrobial Activity of Selected Essential Oils against Staphylococcus spp. Isolated from Human Semen
}

\author{
Miroslava Kačániová ${ }^{1,2, *(\mathbb{D})}$, Margarita Terentjeva ${ }^{3}\left(\mathbb{D}\right.$, Jana Štefániková 4 (D), Jana Žiarovská ${ }^{5}$, \\ Tatsiana Savitskaya ${ }^{6}$, Dmitrij Grinshpan ${ }^{6}$, Przemysław Łukasz Kowalczewski ${ }^{7}$ (D), \\ Nenad Vukovic ${ }^{8}$ and Eva Trrdá ${ }^{9}$ [D
}

1 Department of Fruit Science, Viticulture and Enology, Faculty of Horticulture and Landscape Engineering, Slovak University of Agriculture, Tr. A. Hlinku 2, 94976 Nitra, Slovakia

2 Department of Bioenergetics, Food Analysis and Microbiology, Institute of Food Technology and Nutrition, University of Rzeszow, Cwiklinskiej 1, 35-601 Rzeszow, Poland

3 Institute of Food and Environmental Hygiene, Faculty of Veterinary Medicine, Latvia University of Life Sciences and Technologies, K. Helmana iela 8, LV-3004 Jelgava, Latvia; margarita.terentjeva@llu.lv

4 AgroBioTech Research Centre, Slovak University of Agriculture, Tr. A. Hlinku 2, 94976 Nitra, Slovakia; jana.stefanikova@uniag.sk

5 Department of Plant Genetics and Breeding, Faculty of Agrobiology and Food Resources, Slovak University of Agriculture, Tr. A. Hlinku 2, 94976 Nitra, Slovakia; jana.ziarovska@uniag.sk

6 Research Institute for Physical Chemical Problems, Belarusian State University, Leningradskaya str. 14, 220030 Minsk, Belarus; savitskayaTA@bsu.by (T.S.); Grinshpan@bsu.by (D.G.)

7 Department of Food Technology of Plant Origin, Poznań University of Life Sciences, 31 Wojska Polskiego St., 60-624 Poznań, Poland; przemyslaw.kowalczewski@up.poznan.pl

8 Department of Chemistry, Faculty of Science, University of Kragujevac, P.O. Box 12, 34000 Kragujevac, Serbia; nvchem@yahoo.com

9 Department of Animal Physiology, Faculty of Biotechnology and Food Sciences, Slovak University of Agriculture, Tr. A. Hlinku 2, 94976 Nitra, Slovakia; eva.tvrda@uniag.sk

* Correspondence: miroslava.kacaniova@gmail.com

Received: 10 October 2020; Accepted: 29 October 2020; Published: 31 October 2020

\begin{abstract}
Staphylococcus spp. is not only a commensal bacteria but also a major human pathogen that causes a wide range of clinical infections. Recent evidence suggests that Staphylococcus has the ability to colonize the reproductive system and to affect its structure and functions. The objective of this study was to determine the chemical properties and antibacterial effects of select essential oils (EOs): Amyris balsamifera L., Boswellia carterii Birdw., Canarium luzonicum (Blume) A. Gray, Cinnamomum camphora (L.) J. Presl., Cinnamomum camphora var. linaloolifera Y. Fuita, Citrus x aurantium L., Gaultheria procumbens L., Litsea cubeba (Lour.) Pers., Melaleuca ericifolia Smith., Melaleuca leucadendra L., Pogostemon cablin (Blanco) Benth., Citrus limon (L.) Osbeck, Santalum album L., and Vetiveria zizanoides (L.) Roberty against 50 Staphylococcus spp. cultures isolated from human semen, specifically Staphylococcus aureus, S. capiti, S. epidermidis, S. haemoliticus, and S. hominis. The disc diffusion and broth microdilution methods were used to assess the antimicrobial potential and to determine the minimum inhibitory concentration (MIC) of the selected EOs. The best anti-Staphylococcus activities were found with both methods for the essential oils of C. luzonicum (Blume) A. Gray, A. balsamifera, C. camphora, and P. cabli.
\end{abstract}

Keywords: Staphylococcus spp.; human semen; essential oils; antimicrobial activity; antimicrobial resistance 


\section{Introduction}

As much as fifteen percent of infertility in males are a result of infections of the genitourinary tract [1]. Infections, both chronic and acute, lead to inflammation which compromises proper spermatogenic function [2-4]. This causes alterations in the sperm quality and quantity. Semen contamination occurs from microbiota present in the urinary tract or is transmitted via sexual intercourse [5].

Staphylococcus spp. has been frequently isolated from the reproductive system of men; furthermore, their ability to infect the male reproductive tract has been reported. Staphylococcus spp. may impair the secretory capacity of the epididymis, seminal vesicles, and prostate and may significantly affect sperm quality [6]. Essential oils (EOs) are a rich source of bioactive compounds, with some EOs exhibiting pronounced antimicrobial activity. Many plant parts, such as leaves, seeds, bark, resin, berries, flowers, roots, or fruits, contain EOs [7]. It has been shown that EOs of different plants and parts of the plant differ significantly in chemical composition and antimicrobial properties. Despite significant progress in the research of antimicrobial activity, extraction, and utilization of EOs, field studies on their application on opportunistic and pathogenic microbiota isolated from humans are needed [8]. Previous research suggests that the antimicrobial effect of EOs on human isolates could be used to prevent community- or hospital-acquired infections, which could become a suitable strategy to minimize the spread of antimicrobial resistance and to increase the efficiency of conservative treatment options [7-9].

The strongest antimicrobial activity of the Juniperus communis essential oil was found against S. hominis [10]. Salari et al. [11] used Eucalyptus globulus leaf extract to evaluate its activity on 56 isolates of S. aureus. The EOs extracted from all seven Eucalyptus spp. exhibited antibacterial activity against S. aureus. The best antimicrobial activity of E. globulus was found against S. aureus and S. capiti. In the meantime, Cananga odorata showed the best antimicrobial activity against $S$. hominis [10].

The objective of the present study was to investigate the chemical properties of selected essential oils and their antimicrobial effects against Staphylococcus spp. isolated from human semen.

\section{Results and Discussion}

\subsection{Isolated Species of Staphylococci}

In our study, 96 isolates were identified with mass spectrometry, with 50 isolates receiving a score higher than 2.00. The Staphylococcus spp. strains were Staphylococcus aureus (1 isolate), S. capitis (1 isolate), S. epidermidis (7 isolates), S. haemoliticus (26 isolates), and S. hominis (15 isolates) among the reliably identified isolates. The dendrogram of relatedness of mass spectra of Staphylococcus species is shown in Figure 1.

Two main branches with multiple subbranches can be seen in the constructed dendrogram. The diversity of spectra of all Staphylococcus haemolyticus were obtained as more narrow while the spectra of Staphylococcus epidermis were most diverse in comparison to all other Staphylococcus spp. that were analysed. Staphylococcus capitis and Staphylococcus aureus were assigned to be similar to the Staphylococcus epidermis group according to their protein profiles. A third compact group was created for the mass spectra of Staphylococcus hominis with two isolates that were related to other branches.

Infertility has become a commonly observed clinical diagnosis with infections of the genital tract being frequently identified in patients who undergo assisted reproductive therapy [12].

Infections of the genital tract are caused by microorganisms transmitted from the urinary tract or sexually transmitted as a result of sexual activity. Changes in the morphology and motility of spermatozoa as well as a reduced sperm viability have been identified as a result of the infection [13]. Up to $34.4 \%$ of semen samples were found to be contaminated with microorganisms, predominantly with Staphylococcus spp., Enterococcus, and Escherichia coli [14]. 


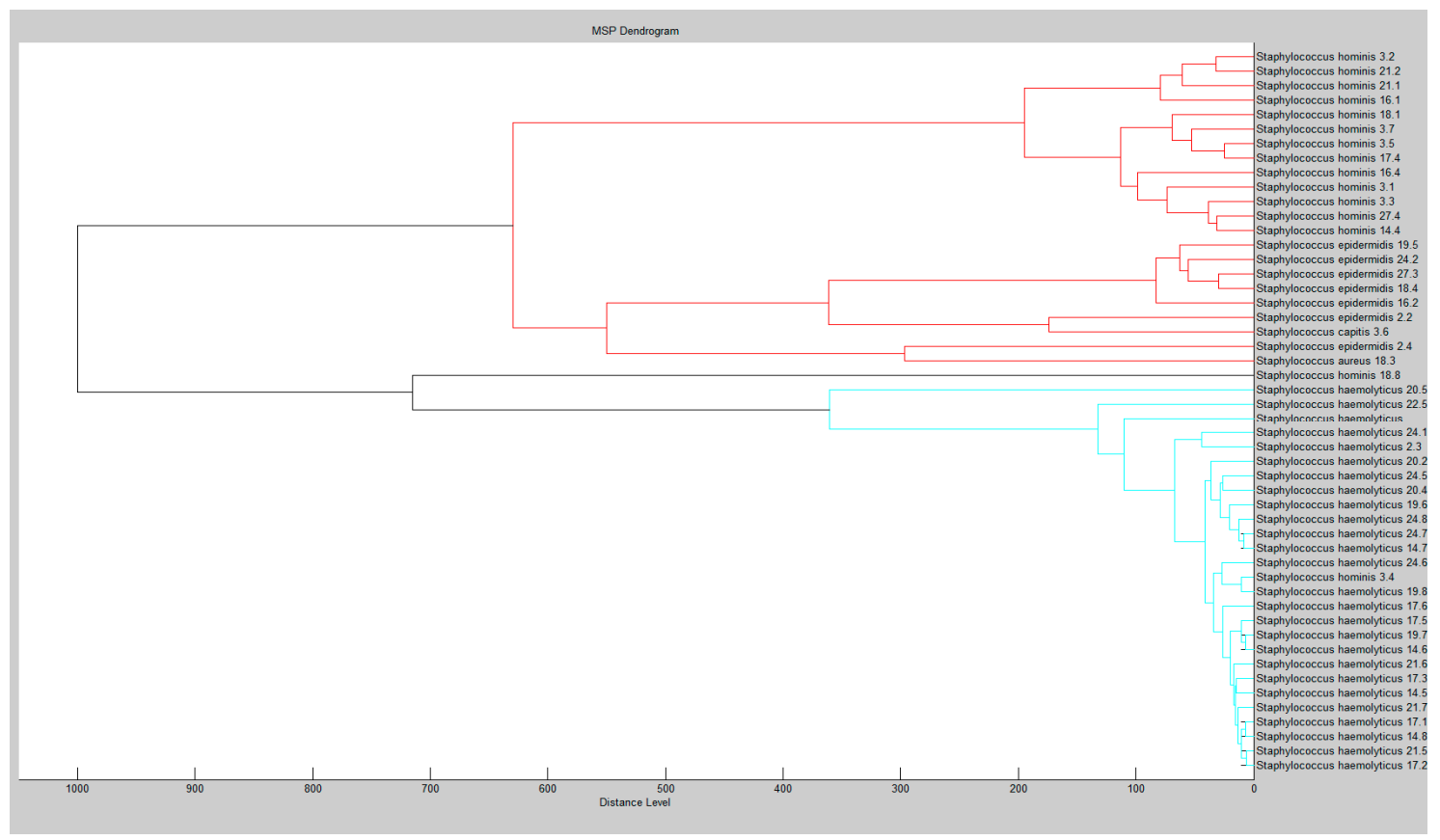

Figure 1. Dendogram of isolated Staphylococcus spp. from human semen constructed with a MALDI-TOF MS Biotyper.

\subsection{Chemical Composition of Essential Oils}

Different factors affecting the chemical composition of EOs. The most prominent endogenous factors are related to anatomical and physiological characteristics of the plants and to biosynthetic pathways of the volatiles, which might change depending on the plant tissue or season; however, it could also be influenced by DNA adaptation. On the other hand, exogenous factors might affect some of the genes responsible for volatiles formation, especially over a long period of time. Such changes may lead to ecotypes or chemotypes within the same plant species [15].

The chemical composition of Amyris balsamifera L. EO is shown in Table 1. The EO was obtained by steam distillation of crushed fresh wood. The presence of 15 chemical components with min $1 \%$ for each were identified. The compounds present in the highest amounts were valerianol $(23.20 \%)$, guaiol $(19.40 \%)$, and 10-epi- $\gamma$-eudesmol $(14.80 \%)$. Different results were found in the study by Klouček et al. [16], where $\alpha$-eudesmol (29.4\%), $\beta$-eudesmol (10.4\%), and valerianol (10.2\%) were the main compounds of the amyris essential oil.

Table 1. Chemical composition of essential oil (\%)*.

\begin{tabular}{cccc}
\hline Essential Oil & Components & RT (min.) & $\begin{array}{c}\text { Percentage of } \\
\text { Components }(\mathbf{n}=\mathbf{3})\end{array}$ \\
\hline & Amorpha-4,11-diene & 24.20 & 2.58 \\
& $\beta$-cadinene & 25.04 & 1.33 \\
Dihydroagarofuran & 26.43 & 1.53 \\
& -dihydroagarofuran & 26.56 & 1.05 \\
& $\alpha$-zingiberene & 27.06 & 2.21 \\
& Cedrene & 28.72 & 4.91 \\
& $\alpha$-curcumen & 28.89 & 2.44 \\
Amyris balsamifera $\mathrm{L}$ Nerolidol & Elemol & 35.64 & 1.57 \\
& $\beta$-eudesmol & 39.25 & 9.62 \\
& $\gamma$-eudesmol & 39.75 & 1.13 \\
& Guaiol & 40.50 & 2.49 \\
& 10-epi- $\gamma$-eudesmol & 40.94 & 19.40 \\
& Valerianol & 41.75 & 14.80 \\
& Drim-7-en-11-ol & 43.16 & 23.20 \\
& & 50.65 & 1.84 \\
\hline
\end{tabular}


Table 1. Cont.

\begin{tabular}{|c|c|c|c|}
\hline Essential Oil & Components & RT (min.) & $\begin{array}{c}\text { Percentage of } \\
\text { Components }(n=3)\end{array}$ \\
\hline \multirow{19}{*}{ Boswelia carterii Birdw. } & Sabinene & 4.33 & 5.51 \\
\hline & 3-Carene & 5.05 & 1.39 \\
\hline & $\alpha$-phellandrene & 5.54 & 2.81 \\
\hline & $\beta$-myrcene & 5.65 & 3.91 \\
\hline & D-limonene & 6.68 & 26.40 \\
\hline & 4-Thujanol & 6.70 & 1.49 \\
\hline & prehnitene & 9.25 & 8.65 \\
\hline & Copaene & 17.96 & 1.59 \\
\hline & Bornyl acetate & 21.6 & 1.00 \\
\hline & 2-methylene-4,8,8-trimethyl-4-vinyl-bicyclo[5.2.0]nonane & 21.93 & 7.83 \\
\hline & Farnesol & 24.6 & 2.24 \\
\hline & trans-verbenol & 25.65 & 1.55 \\
\hline & $\beta$-selinene & 26.39 & 2.18 \\
\hline & $\gamma$-selinene & 26.63 & 1.24 \\
\hline & $\gamma$-cadinene & 28.02 & 1.67 \\
\hline & $\delta$-cadinene & 28.12 & 2.05 \\
\hline & Carveol & 31.32 & 1.03 \\
\hline & Caryophyllene oxide & 35.28 & 2.41 \\
\hline & tau-cadinol & 41.74 & 2.13 \\
\hline \multirow{12}{*}{$\begin{array}{l}\text { Canarium luzonicum } \\
\text { (Blume) A. Gray }\end{array}$} & $\beta$-Phellandrene & 4.34 & 4.54 \\
\hline & $\alpha$-Phellandrene & 5.54 & 12.20 \\
\hline & D-limonene & 6.68 & 36.40 \\
\hline & cis-Sabinene & 6.90 & 3.06 \\
\hline & o-cymene & 9.25 & 3.35 \\
\hline & $\alpha$-terpinolen & 9.75 & 1.59 \\
\hline & Terpinen-4-ol & 22.71 & 1.15 \\
\hline & $\alpha$-terpineol & 26.41 & 3.83 \\
\hline & Elemol & 39.25 & 16.70 \\
\hline & 10-epi- $\gamma$-eudesmol & 41.75 & 1.59 \\
\hline & Rosifoliol & 43.27 & 1.08 \\
\hline & Elemicin & 43.73 & 9.59 \\
\hline \multirow{6}{*}{$\begin{array}{l}\text { Cinnamomum camphora } \\
\text { (L.) J. Presl. }\end{array}$} & Sabinene & 4.33 & 6.07 \\
\hline & $\beta$-pinene & 5.64 & 1.70 \\
\hline & D-limonene & 6.68 & 25.90 \\
\hline & 1,8-cineol & 7.07 & 44.90 \\
\hline & $\gamma$-terpinene & 8.35 & 1.43 \\
\hline & o-cymene & 9.25 & 11.70 \\
\hline \multirow{2}{*}{$\begin{array}{l}\text { Cinnamomum caphora var. } \\
\text { linaloolifera } \text { Y. Fuita }\end{array}$} & 1,2-epoxylinalool & 16.46 & 1.27 \\
\hline & Linalool & 21.32 & 96.99 \\
\hline \multirow{10}{*}{ Citrus $x$ aurantium L. } & $\beta$-myrcene & 5.65 & 2.32 \\
\hline & D-limonene & 6.68 & 1.57 \\
\hline & 1,8-cineole & 7.07 & 2.70 \\
\hline & $\beta$-ocimene & 8.75 & 2.39 \\
\hline & Linalyl acetate & 21.14 & 63.4 \\
\hline & Caryophyllene & 21.92 & 1.34 \\
\hline & $\alpha$-terpineol & 26.41 & 8.84 \\
\hline & Neryl acetate & 27.51 & 3.77 \\
\hline & Geranyl acetate & 28.64 & 6.02 \\
\hline & Geraniol & 30.28 & 5.31 \\
\hline Gaultheria procumbens L. & Methyl salicylate & 28.68 & 98.00 \\
\hline \multirow{12}{*}{ Litsea cubeba (Lour.) Pers. } & Sabinene & 4.33 & 2.20 \\
\hline & D-limonene & 6.68 & 14.00 \\
\hline & 1,8-cineole & 7.07 & 1.62 \\
\hline & 6-methyl-5-hepten-2-one & 12.11 & 1.35 \\
\hline & Citronellal & 17.86 & 1.00 \\
\hline & Linalool & 21.32 & 1.82 \\
\hline & Caryophyllene & 21.92 & 1.85 \\
\hline & (Z)-citral & 25.56 & 31.00 \\
\hline & $\alpha$-terpineol & 26.41 & 1.06 \\
\hline & (E)-citral & 27.51 & 35.2 \\
\hline & trans-geraniol & 32.01 & 1.35 \\
\hline & nerolic acid & 45.74 & 1.13 \\
\hline
\end{tabular}


Table 1. Cont.

\begin{tabular}{|c|c|c|c|}
\hline Essential Oil & Components & RT (min.) & $\begin{array}{c}\text { Percentage of } \\
\text { Components }(n=3)\end{array}$ \\
\hline \multirow{13}{*}{ Melaleuca leucadendron L. } & D-limonene & 6.68 & 8.12 \\
\hline & 1,8-Cineole & 7.07 & 49.20 \\
\hline & $\gamma$-terpinen & 8.35 & 2.91 \\
\hline & o-cymene & 9.25 & 3.16 \\
\hline & $\alpha$-terpinolen & 9.75 & 1.24 \\
\hline & Linalyl acetate & 21.14 & 1.13 \\
\hline & Caryophyllene & 21.92 & 5.65 \\
\hline & 2,4-dihydroxy-2-methylpentane & 24.12 & 4.11 \\
\hline & $\mathrm{Z}, \mathrm{Z}, \mathrm{Z}-1,5,9,9$-tetramethyl-1,4,7,-cycloundecatriene & 24.61 & 2.91 \\
\hline & $\alpha$-terpineol acetate & 26.07 & 1.84 \\
\hline & $\alpha$-terpineol & 26.41 & 9.92 \\
\hline & $\alpha$-selinene & 26.64 & 2.09 \\
\hline & Globulol & 38.72 & 1.90 \\
\hline \multirow{14}{*}{ Melaleuca ericifolia Smith. } & D-limonene & 6.68 & 2.97 \\
\hline & 1,8-cineol & 7.07 & 23.10 \\
\hline & $\gamma$-terpinene & 8.35 & 2.63 \\
\hline & o-cymene & 9.25 & 3.64 \\
\hline & 1,2-epoxylinalool & 16.46 & 2.03 \\
\hline & Ethyl & & \\
\hline & $\begin{array}{l}\text { 2-(5-methyl-5-vinyltetrahydrofuran-2-yl)propan-2-yl } \\
\text { carbonate }\end{array}$ & 17.61 & 1.54 \\
\hline & $\beta$-linalool & 21.24 & 36.70 \\
\hline & 2-methylene-4,8,8-trimethyl-4-vinyl-bicyclo[5.2.0]nonane & 21.93 & 1.17 \\
\hline & Alloaromadendrene & 22.40 & 4.73 \\
\hline & Terpinen-4-ol & 22.71 & 2.62 \\
\hline & Aromadendrene & 23.75 & 1.86 \\
\hline & Leden & 25.73 & 1.07 \\
\hline & $\alpha$-terpineol & 26.41 & 4.98 \\
\hline \multirow{10}{*}{$\begin{array}{l}\text { Pogostemon cabli (Blanco) } \\
\text { Benth. }\end{array}$} & Patchoulene & 17.51 & 3.28 \\
\hline & $\alpha$-guaien & 22.10 & 18.10 \\
\hline & $\alpha$-patchoulene & 23.23 & 6.09 \\
\hline & 1,1,4a-Trimethyl-5,6-dimethylenedecahydronaphthalene & 23.33 & 7.88 \\
\hline & $\gamma$-patchoulene & 23.88 & 1.10 \\
\hline & Aciphyllene & 26.11 & 3.53 \\
\hline & $\gamma$-guajene & 26.69 & 18.20 \\
\hline & Patchouli alcohol & 41.48 & 27.30 \\
\hline & Pogostol & 42.74 & 2.65 \\
\hline & Dhelqangin & 51.98 & 2.34 \\
\hline \multirow{8}{*}{ Citrus limon (L.) Osbeck } & (E)-citral & 27.51 & 2.44 \\
\hline & Sabinene & 4.33 & 3.45 \\
\hline & $\beta$-myrcene & 5.65 & 2.42 \\
\hline & D-limonene & 6.68 & 67.10 \\
\hline & $\alpha$-terpinolen & 9.75 & 14.20 \\
\hline & $\alpha$-bergamotene & 21.89 & 1.33 \\
\hline & Neral & 25.45 & 1.50 \\
\hline & $\beta$-bisabolene & 27.22 & 1.30 \\
\hline \multirow{8}{*}{ Santalum album L. } & $\alpha$-santalene & 21.31 & 5.03 \\
\hline & $\alpha$-bergamotene & 21.89 & 9.68 \\
\hline & $\alpha$-santalal & 41.14 & 2.54 \\
\hline & $\alpha$-santalol & 41.69 & 59.00 \\
\hline & $\beta$-santalol & 50.36 & 9.02 \\
\hline & Lanceol & 51.12 & 1.93 \\
\hline & $(E)$-nuciferol & 51.87 & 1.74 \\
\hline & 7-(5-hexynyl)-tricyclo[4.2.2.0(2,5)]dec-7-ene & 52.26 & 1.38 \\
\hline
\end{tabular}


Table 1. Cont.

\begin{tabular}{|c|c|c|c|}
\hline Essential Oil & Components & RT (min.) & $\begin{array}{c}\text { Percentage of } \\
\text { Components }(n=3)\end{array}$ \\
\hline \multirow{27}{*}{$\begin{array}{c}\text { Vetiveria zizanoides (L.) } \\
\text { Roberty }\end{array}$} & 3,3,5,6,8,8-Hexamethyltricyclo[5.1.0.0(2,4)]oct-5-ene & 18.88 & 1.02 \\
\hline & Tricyclo[6.3.0.0(1,5)]undec-2-en-4-one, 5,9-dimethyl & 20.76 & 2.00 \\
\hline & 1,2,4,5-tetraethylbenzene & 21.32 & 4.39 \\
\hline & $\alpha$-muurolene & 25.59 & 1.89 \\
\hline & $\alpha$-cadinene & 25.78 & 1.74 \\
\hline & Selina-5,11-diene & 26.16 & 1.50 \\
\hline & $\delta$-cadinene & 26.64 & 1.57 \\
\hline & $\alpha$-vetispirene & 27.16 & 1.77 \\
\hline & $\beta$-vetispirene & 27.42 & 2.03 \\
\hline & $\gamma$-vetivenene & 30.19 & 3.30 \\
\hline & $\beta$-vetivenene & 31.27 & 7.42 \\
\hline & Valencen & 32.39 & 2.19 \\
\hline & 3,5,11-Eudesmatriene & 34.91 & 1.65 \\
\hline & 7,7-dichlorobicyclo[3.2.0]hept-2-en-6-one & 41.06 & 1.22 \\
\hline & $\gamma$-himachalene & 41.31 & 2.63 \\
\hline & Ziza-6(13)-en-12-al & 41.99 & 1.02 \\
\hline & Khusiol & 45.27 & 1.12 \\
\hline & $\beta$-guaiene & 45.58 & 4.43 \\
\hline & Cyclocopacamphenol & 46.03 & 1.66 \\
\hline & Zizanol & 47.64 & 1.03 \\
\hline & (E)-isovalencenal & 47.76 & 1.37 \\
\hline & Vetiselinenol & 48.57 & 1.41 \\
\hline & Vetiverol & 50.15 & 2.22 \\
\hline & Khusenol & 50.68 & 5.24 \\
\hline & Vetiverone & 51.09 & 3.02 \\
\hline & $\beta$-costol & 52.11 & 3.52 \\
\hline & Khusenic acid & 63.32 & 4.17 \\
\hline
\end{tabular}

Note: ${ }^{*}$ listed are the components that represented $\min .1 \%$. Values represent means of three replicate determinations (maximum relative standard deviation $\pm 5 \%$ ).

The chemical composition of Boswelia carterii Birdw. EO is given in Table 1. The EO was obtained by steam distillation of hand-collected resin. Nineteen chemical components with min $1 \%$ were identified. D-limonene (26.40\%) and prehnitene (prehnitol, $8.65 \%$ ) were the main compounds, which is in agreement with Camarda et al. [17].

The chemical composition of Canarium luzonicum (Blume) A. Gray EO is shown in Table 1. The EO was collected by steam distillation of resin. The presence of 12 chemical components with $\min 1 \%$ was found. The main compounds were D-limonene (36.40\%) and elemol $(16.70 \%)$, similar to the report of Villanueva et al. [18].

The chemical composition of Cinnamomum camphora (L.) J. Presl. EO is provided in Table 1. The EO was obtained by redistillation of wood and branches by steam, so-called white fraction, which does not contain safrole. Six chemical components with $\min 1 \%$ were found. The dominant constituents were 1,8-cineol (eucalyptol, $44.90 \%)$, D-limonene (25.90\%), and o-cymene (11.70\%). A previous study on the EO from fruits in the Guizhou province reported D-camphor (26.10\%), 1,8-cineole (19.90\%), linalool $(9.20 \%), \alpha$-terpineol $(7.20 \%)$, and limonene (5.30\%) [19]. The main constituents in the sample from Jiangxi were D-camphor (42.80\%), 1,8-cineole (24.80\%), $\alpha$-terpineol (8.70\%), and $\beta$-pinene (5.80\%) [20].

The chemical composition of Cinnamomum caphora var. linaloolifera Y. Fuita EO is presented in Table 1. The EO was acquired by steam distillation of leaves. The main compound was linalool $(96.99 \%)$. Linalool was found to be the major constituent of C. caphora var. linaloolifera leaf oil $(95.00 \%)$, with no other compounds present at a level of more than $1 \%$ [21].

The chemical composition of Citrus $x$ aurantium L. EO is given in Table 1 . The EO was obtained by distillation of fresh leaves. The presence of 11 chemical components with min $1 \%$ was recorded. The main compounds were linalyl acetate $(63.40 \%)$ and $\alpha$-terpineol ( $p$-menth-1-en-8-ol, $8.84 \%)$, with linalool and linalyl acetate in leaves and limonene being found in previous studies [22,23].

The chemical composition of Gaultheria procumbens L. EO is presented in Table 1. The EO was acquired by distillation of freshly fermented fresh leaves. Methyl salicylate $(98.00 \%)$ was the main compound which is in agreement with a previous report [24] 
The chemical composition of Litsea cubeba (Lour.) Pers. EO is shown in Table 1. The EO was obtained by distillation of fruits. The presence of 11 chemical components with min $1 \%$ was found: (E)-citral ((F)-geranial and (E)-neral, 35.20\%), (Z)-citral ((Z)-neral, 31.00\%), and D-limonene (14.00\%). Our results are in agreement with Thielmann and Muranyi [25], who stated that citral and limonene were the major components of L. cubeba EO extracted from fruits.

The chemical composition of Melaleuca leucadendron L. EO is given in Table 1. The EO was obtained by steam distillation of young shoots and leaves. The presence of 11 chemical components with min $1 \%$ was recorded. The main compounds were 1,8-cineol (eucalyptol, $49.20 \%$ ) and $\alpha$-terpineol $(9.92 \%)$, which is line with previously reported 1,8-cineole (44.8-60.2\%), $\alpha$-terpineol (5.93-12.5\%), D-limonene (4.45-8.85\%), and $\beta$-caryophyllene (3.78-7.64\%) [26].

The chemical composition of Melaleuca ericifolia Smith. EO is provided in Table 1. The EO was collected by steam distillation of branches. The presence of 13 chemical components with min $1 \%$ was observed. The main compounds were $\beta$-linalool (linalyl alcohol, 36.70\%) and 1,8-cineol (eucalyptol, $23.10 \%$ ). The EO from the leaves of M. leucadendra from Vietnam were found to be rich in $\alpha$-eudesmol (17.6-21.2\%) and guaiol (10.9-12.5\%), and linalool was present in smaller concentrations (4.9-5.1\%) [27]. Other studies indicated that 1,8-cineole was the major compound of M. leucadendron oil [28-30].

The chemical composition of Pogostemon cabli (Blanco) Benth. EO is given in Table 1. The EO was obtained by distillation of fermented leaves with steam, followed by maturation of the EO over time. Ten chemical components were present at min $1 \%$, including patchouli alcohol $(27.30 \%), \gamma$-guajene ( $\alpha$-bulnesene, $18.20 \%$ ), and $\alpha$-guaien $(18.10 \%)$. The major components of the oil were reported to be acetophenone (51.00\%), $\beta$-pinene (5.30\%), (E)-nerolidol (5.40\%), and patchouli alcohol (14.00\%) [31].

The chemical composition of Citrus limon (L.) Osbeck EO is displayed in Table 1. The EO was acquired by cold pressing fresh fruit. The presence of 8 chemical components that represented min $1 \%$ was recorded. D-limonene $(67.10 \%)$ and $p$-mentha-1,4(8)-diene (iso-terpinene and $\alpha$-terpinolene, $14.20 \%$ ) were the main compounds while limonene $(55.40 \%)$, neral $(10.40 \%)$, trans-verbenol $(6.43 \%)$, and decanal $(3.25 \%)$ were found to be the main components among 43 identified compounds in the EO of this fruit in India [32].

The chemical composition of Santalum album L. EO is given in Table 1. The EO was obtained by steam distillation of crushed wood. Twelve chemical components were identified with a min $1 \%$. The main compounds were $\alpha$-santalol (59.00\%), $\alpha$-bergamotene (9.68\%), and $\beta$-santalol (9.02\%). Among those, $\alpha$ - and $\beta$-santalol, which accounted for $19.60 \%$ and $16.00 \%$, respectively, were identified in India, and cis- $\alpha$-santalol was recorded in the EOs from Sri Lanka $[33,34]$.

The chemical coposition of Vetiveria zizanoides (L.) Roberty EO is presented in Table 1. The EO was obtained by steam distillation of sun-dried roots. The analysis indicated the presence of 28 chemical components at $\min 1 \%$. The main compounds were $\beta$-vetivenene $(7.42 \%)$ and khusenol $(5.24 \%)$. David et al., 2009, analyzed oils extracted with carbon dioxide-expanded ethanol and found valerenol $(18.50 \%)$, valerenal $(10.20 \%)$, and $\beta$-cadinene $(6.23 \%)$ to be the most common compounds out of a total of 23 molecules identified. Interestingly, 48 more components were found in oils processed with conventional hydrodistillation [35].

\subsection{Antibacterial Effect of Antimicrobials}

In this study, 50 isolates of Staphylococcus spp. acquired from human semen were tested for antimicrobial resistance (Table 2) against chloramphenicol, tetracycline, tigecycline, and tobramycin, and the results were interpreted according to the European Committee on Antimicrobial Susceptibility Testing (EUCAST) guidelines [36]. In total, 37 (74\%) isolates were resistant while $13(26 \%)$ isolates were sensitive to chloramphenicol. All tested isolates were sensitive to tetracycline and tigecycline. Resistance to tobramycin was identified in the case of 32 isolates, while 10 were sensitive and 8 were intermediately resistant to tobramycin. 
Table 2. Antimicrobial resistance of Staphylococcus spp.

\begin{tabular}{|c|c|c|c|c|}
\hline Name of Bacteria/AMB & $\mathrm{C}$ & TET & TIG & TOB \\
\hline Stapyloccocus aureus 18.3 & $S$ & $\mathrm{~S}$ & $S$ & $\mathrm{~S}$ \\
\hline Staphyloccous capitis 3.6 & $\mathrm{R}$ & $S$ & S & $\mathrm{I}$ \\
\hline Staphylococcus epidermidis 2.2 & $\mathrm{R}$ & S & S & $\mathrm{R}$ \\
\hline Staphylococcus epidermidis 2.4 & $S$ & S & S & $\mathrm{R}$ \\
\hline Staphylococcus epidermidis 16.2 & $S$ & $S$ & S & $\mathrm{R}$ \\
\hline Staphylococcus epidermidis 18.4 & $\mathrm{R}$ & S & S & $\mathrm{R}$ \\
\hline Staphylococcus epidermidis 19.5 & $\mathrm{~S}$ & $\mathrm{~S}$ & S & $\mathrm{R}$ \\
\hline Staphylococcus epidermidis 24.2 & $\mathrm{R}$ & S & S & $\mathrm{I}$ \\
\hline Staphylococcus epidermidis 27.3 & $\mathrm{R}$ & $\mathrm{S}$ & S & $\mathrm{R}$ \\
\hline Staphylococcus haemoliticus 2.3 & $\mathrm{R}$ & S & $S$ & $\mathrm{R}$ \\
\hline Staphylococcus haemoliticus 14.5 & $\mathrm{R}$ & $S$ & S & $\mathrm{R}$ \\
\hline Staphylococcus haemoliticus 14.6 & $\mathrm{R}$ & $\mathrm{S}$ & S & $\mathrm{R}$ \\
\hline Staphylococcus haemoliticus 14.7 & S & S & S & $\mathrm{R}$ \\
\hline Staphylococcus haemoliticus 14.8 & $\mathrm{R}$ & $S$ & S & $\mathrm{R}$ \\
\hline Staphylococcus haemoliticus 17.1 & $\mathrm{R}$ & S & S & $\mathrm{R}$ \\
\hline Staphylococcus haemoliticus 17.2 & $\mathrm{R}$ & $\mathrm{S}$ & S & $\mathrm{R}$ \\
\hline Staphylococcus haemoliticus 17.3 & $\mathrm{R}$ & S & S & $\mathrm{R}$ \\
\hline Staphylococcus haemoliticus 17.5 & S & S & S & $\mathrm{R}$ \\
\hline Staphylococcus haemoliticus 17.6 & $\mathrm{R}$ & $\mathrm{S}$ & S & $S$ \\
\hline Staphylococcus haemoliticus 19.7 & $\mathrm{R}$ & S & S & $\mathrm{R}$ \\
\hline Staphylococcus haemoliticus 19.8 & $S$ & $S$ & S & $\mathrm{R}$ \\
\hline Staphylococcus haemoliticus 20.2 & $S$ & $S$ & $S$ & $\mathrm{R}$ \\
\hline Staphylococcus haemoliticus 20.4 & $\mathrm{R}$ & $S$ & S & $\mathrm{I}$ \\
\hline Staphylococcus haemoliticus 20.5 & $\mathrm{R}$ & S & S & $\mathrm{R}$ \\
\hline Staphylococcus haemoliticus 21.5 & $\mathrm{R}$ & S & S & $\mathrm{S}$ \\
\hline Staphylococcus haemoliticus 21.6 & $S$ & $\mathrm{~S}$ & S & $\mathrm{I}$ \\
\hline Staphylococcus haemoliticus 21.7 & $\mathrm{R}$ & S & S & $\mathrm{R}$ \\
\hline Staphylococcus haemoliticus 22.5 & $\mathrm{R}$ & $S$ & S & $\mathrm{R}$ \\
\hline Staphylococcus haemoliticus 24.1 & $\mathrm{R}$ & S & S & S \\
\hline Staphylococcus haemoliticus 24.2 & $\mathrm{R}$ & $\mathrm{S}$ & S & $\mathrm{R}$ \\
\hline Staphylococcus haemoliticus 24.5 & $\mathrm{R}$ & S & S & S \\
\hline Staphylococcus haemoliticus 24.6 & $S$ & S & S & I \\
\hline Staphylococcus haemoliticus 24.7 & $\mathrm{R}$ & $\mathrm{S}$ & S & $\mathrm{R}$ \\
\hline Staphylococcus haemoliticus 24.8 & $\mathrm{R}$ & $\mathrm{S}$ & S & $\mathrm{R}$ \\
\hline Staphylococcus haemoliticus & $\mathrm{R}$ & S & S & S \\
\hline Staphylococus hominis 3.1 & $S$ & S & $S$ & $\mathrm{R}$ \\
\hline Staphylococus hominis 3.2 & $\mathrm{R}$ & $\mathrm{S}$ & S & $\mathrm{R}$ \\
\hline Staphylococus hominis 3.3 & $\mathrm{R}$ & S & S & $\mathrm{I}$ \\
\hline Staphylococus hominis 3.4 & $\mathrm{R}$ & $S$ & S & $\mathrm{R}$ \\
\hline Staphylococus hominis 3.5 & $S$ & $S$ & S & $\mathrm{R}$ \\
\hline Staphylococus hominis 3.7 & $\mathrm{R}$ & $\mathrm{S}$ & S & $S$ \\
\hline Staphylococus hominis 14.4 & $\mathrm{R}$ & $S$ & S & $\mathrm{R}$ \\
\hline Staphylococus hominis 16.1 & $\mathrm{R}$ & $S$ & $S$ & $S$ \\
\hline Staphylococus hominis 16.4 & $S$ & $\mathrm{~S}$ & S & $\mathrm{R}$ \\
\hline Staphylococus hominis 17.4 & $\mathrm{R}$ & S & $S$ & $\mathrm{R}$ \\
\hline Staphylococus hominis 18.1 & $\mathrm{R}$ & $S$ & $S$ & $\mathrm{R}$ \\
\hline Staphylococus hominis 18.8 & $\mathrm{R}$ & S & S & I \\
\hline Staphylococus hominis 21.1 & $\mathrm{R}$ & $\mathrm{S}$ & S & $S$ \\
\hline Staphylococus hominis 21.2 & $\mathrm{R}$ & $S$ & $S$ & $\mathrm{I}$ \\
\hline Staphylococus hominis 27.4 & $\mathrm{R}$ & $S$ & $S$ & $S$ \\
\hline
\end{tabular}

Note: C—chloramphenicol, TET—-tetracycline, TIG—tigecycline, TO-tobramycin, R-resistant, S—sensitive, I-intermediate.

Chloramphenicol is a broad spectrum antimicrobial which is active against gram-positive as well as gram-negative bacteria $[37,38]$. Because of chrolamphenicol toxicity and its application for life-treatening conditions, highly phenicol-resistant $S$. aureus strains of human origin have become a pressing area of scientific interest [39]. Resistance to tetracyclines is common as a result of their 
broad implementation in human and veterinary medicine. Furthermore, antimicrobial resistance to tetracycline has emerged in plants as well $[40,41]$. Resistance to tetracycline is encoded by genetic determinants and is fairly common in bacteria [42]. Tigecycline activity in vitro was observed against gram-positive and gram-negative microorganisms, such as S. aureus, Enterococcus spp., S. pneumoniae, Haemophilus influenzae, Moraxella catarrhalis, Neisseria gonorrhoeae, N. peptostreptococci, Clostridium spp., Enterobacteriaceae, and Bacteroides spp. [43,44]. It must be noted that differences in the antimicrobial resistance rates against gentamicin and tobramycin were found for S. aureus and P. aeruginosa across Europe [45].

\subsection{Antimicrobial Assay}

The antibacterial activities of 14 EOs against 50 Staphylococcus spp. isolates were determined with disc diffusion and broth dilution methods (Tables 3-6). The antimicrobial properties of the assessed oils exhibited broad variations.

Table 3. Antimicrobial activity of essential oils (EOs) with disc diffusion method in $\mathrm{mm}$.

\begin{tabular}{|c|c|c|c|c|c|c|c|}
\hline Isolate/EOs & 1. & 2. & 3. & 4. & 5. & 6. & 7. \\
\hline Stapyloccocus aureus 18.3 & $16.50 \pm 1.32$ & $11.00 \pm 1.00$ & $21.33 \pm 1.15$ & $7.67 \pm 0.58$ & $24.67 \pm 0.58$ & $14.67 \pm 0.58$ & $7.33 \pm 0.58$ \\
\hline Staphyloccous capitis 3.6 & $12.33 \pm 0.58$ & $8.67 \pm 0.58$ & $24.67 \pm 0.58$ & $4.67 \pm 0.58$ & $14.67 \pm 0.58$ & $7.67 \pm 0.58$ & $8.33 \pm 0.58$ \\
\hline Staphylococcus epidermidis 2.2 & $9.33 \pm 0.58$ & $12.67 \pm 0.58$ & $20.33 \pm 0.58$ & $9.00 \pm 1.00$ & $8.33 \pm 0.58$ & $15.33 \pm 0.58$ & $5.67 \pm 0.58$ \\
\hline Staphylococcus epidermidis 2.4 & $8.67 \pm 0.58$ & $11.67 \pm 0.58$ & $22.33 \pm 0.58$ & $7.67 \pm 0.58$ & $7.33 \pm 0.58$ & $15.00 \pm 0.00$ & $4.67 \pm 0.58$ \\
\hline Staphylococcus epidermidis 16.2 & $11.67 \pm 0.58$ & $12.67 \pm 0.58$ & $21.67 \pm 0.58$ & $4.67 \pm 0.58$ & $7.67 \pm 0.58$ & $16.33 \pm 0.58$ & $5.67 \pm 0.58$ \\
\hline Staphylococcus epidermidis 18.4 & $10.67 \pm 0.58$ & $13.33 \pm 1.15$ & $19.67 \pm 0.58$ & $5.33 \pm 0.58$ & $8.33 \pm 0.58$ & $15.67 \pm 1.15$ & $5.50 \pm 0.50$ \\
\hline Staphylococcus epidermidis 19.5 & $14.67 \pm 0.58$ & $12.67 \pm 0.58$ & $20.33 \pm 0.58$ & $4.67 \pm 0.58$ & $6.33 \pm 1.15$ & $16.67 \pm 0.58$ & $5.00 \pm 1.00$ \\
\hline Staphylococcus epidermidis 24.2 & $11.33 \pm 1.15$ & $11.67 \pm 1.53$ & $19.67 \pm 0.58$ & $6.33 \pm 0.58$ & $7.33 \pm 1.15$ & $16.00 \pm 1.00$ & $4.33 \pm 0.58$ \\
\hline Staphylococcus epidermidis 27.3 & $11.67 \pm 1.53$ & $11.67 \pm 1.15$ & $20.33 \pm 1.15$ & $5.67 \pm 0.58$ & $6.67 \pm 0.58$ & $17.33 \pm 0.58$ & $4.67 \pm 0.58$ \\
\hline Staphylococcus haemoliticus 2.3 & $8.67 \pm 0.58$ & $8.33 \pm 0.58$ & $20.67 \pm 1.15$ & $4.67 \pm 0.58$ & $4.67 \pm 0.58$ & $10.33 \pm 0.58$ & $5.67 \pm 0.58$ \\
\hline Staphylococcus haemoliticus 14.5 & $7.67 \pm 0.58$ & $8.67 \pm 1.15$ & $18.33 \pm 0.58$ & $6.33 \pm 0.58$ & $5.67 \pm 0.58$ & $8.67 \pm 0.58$ & $5.33 \pm 1.15$ \\
\hline Staphylococcus haemoliticus 14.6 & $11.00 \pm 1.00$ & $8.67 \pm 1.15$ & $19.67 \pm 0.58$ & $7.67 \pm 0.58$ & $4.67 \pm 0.58$ & $8.33 \pm 0.58$ & $6.33 \pm 1.15$ \\
\hline Staphylococcus haemoliticus 14.7 & $7.00 \pm 1.00$ & $7.67 \pm 0.58$ & $20.33 \pm 0.58$ & $8.33 \pm 0.58$ & $4.67 \pm 1.15$ & $7.67 \pm 1.15$ & $4.67 \pm 0.58$ \\
\hline Staphylococcus haemoliticus 14.8 & $11.00 \pm 1.00$ & $8.67 \pm 0.58$ & $18.67 \pm 0.58$ & $9.33 \pm 1.15$ & $5.33 \pm 0.58$ & $8.33 \pm 0.58$ & $6.00 \pm 1.00$ \\
\hline Staphylococcus haemoliticus 17.1 & $10.67 \pm 1.15$ & $10.67 \pm 1.15$ & $18.33 \pm 0.58$ & $6.33 \pm 1.15$ & $5.67 \pm 0.58$ & $6.67 \pm 0.58$ & $4.67 \pm 0.58$ \\
\hline Staphylococcus haemoliticus 17.2 & $9.67 \pm 1.15$ & $10.67 \pm 0.58$ & $17.33 \pm 0.58$ & $5.33 \pm 0.58$ & $6.67 \pm 0.58$ & $7.33 \pm 0.58$ & $5.67 \pm 1.15$ \\
\hline Staphylococcus haemoliticus 17.3 & $9.00 \pm 1.00$ & $8.67 \pm 0.58$ & $18.33 \pm 0.58$ & $5.33 \pm 0.58$ & $6.33 \pm 1.15$ & $8.33 \pm 0.58$ & $5.33 \pm 0.58$ \\
\hline Staphylococcus haemoliticus 17.5 & $13.33 \pm 1.15$ & $8.67 \pm 0.58$ & $17.67 \pm 1.15$ & $6.33 \pm 0.58$ & $7.33 \pm 0.58$ & $6.33 \pm 1.15$ & $5.67 \pm 0.58$ \\
\hline Staphylococcus haemoliticus 17.6 & $11.33 \pm 1.15$ & $9.33 \pm 0.58$ & $20.33 \pm 0.58$ & $5.67 \pm 1.15$ & $6.33 \pm 0.58$ & $5.67 \pm 0.58$ & $5.00 \pm 1.00$ \\
\hline Staphylococcus haemoliticus 19.7 & $14.33 \pm 0.58$ & $7.67 \pm 0.58$ & $18.33 \pm 1.15$ & $6.00 \pm 1.73$ & $7.67 \pm 0.58$ & $7.33 \pm 0.58$ & $4.67 \pm 0.58$ \\
\hline Staphylococcus haemoliticus 19.8 & $13.67 \pm 1.53$ & $10.67 \pm 0.58$ & $18.67 \pm 1.15$ & $5.67 \pm 1.15$ & $7.33 \pm 0.58$ & $8.67 \pm 0.58$ & $6.33 \pm 0.58$ \\
\hline Staphylococcus haemoliticus 20.2 & $9.00 \pm 1.00$ & $10.67 \pm 1.15$ & $20.33 \pm 1.15$ & $5.67 \pm 0.58$ & $5.67 \pm 0.58$ & $7.33 \pm 0.58$ & $4.67 \pm 1.15$ \\
\hline Staphylococcus haemoliticus 20.4 & $10.33 \pm 0.58$ & $8.67 \pm 0.58$ & $19.33 \pm 1.15$ & $5.00 \pm 1.00$ & $6.33 \pm 0.58$ & $7.67 \pm 1.15$ & $5.67 \pm 1.15$ \\
\hline Staphylococcus haemoliticus 20.5 & $13.00 \pm 1.00$ & $9.00 \pm 1.00$ & $18.33 \pm 1.15$ & $7.33 \pm 0.58$ & $5.33 \pm 0.58$ & $7.67 \pm 0.58$ & $6.00 \pm 1.00$ \\
\hline Staphylococcus haemoliticus 21.5 & $14.33 \pm 0.58$ & $8.33 \pm 0.58$ & $20.33 \pm 0.58$ & $7.67 \pm 0.58$ & $6.00 \pm 1.00$ & $8.33 \pm 0.58$ & $5.83 \pm 0.29$ \\
\hline Staphylococcus haemoliticus 21.6 & $9.33 \pm 0.58$ & $9.67 \pm 1.15$ & $18.67 \pm 0.58$ & $7.33 \pm 0.58$ & $6.67 \pm 1.15$ & $7.67 \pm 0.58$ & $6.17 \pm 0.29$ \\
\hline Staphylococcus haemoliticus 21.7 & $8.33 \pm 0.58$ & $10.33 \pm 0.58$ & $17.67 \pm 0.58$ & $6.67 \pm 0.58$ & $4.67 \pm 1.15$ & $6.33 \pm 0.58$ & $5.17 \pm 0.29$ \\
\hline Staphylococcus haemoliticus 22.5 & $7.33 \pm 0.58$ & $8.33 \pm 0.58$ & $17.33 \pm 1.15$ & $5.67 \pm 0.58$ & $4.67 \pm 0.58$ & $6.33 \pm 1.15$ & $4.33 \pm 0.58$ \\
\hline Staphylococcus haemoliticus 24.1 & $11.67 \pm 0.58$ & $10.33 \pm 0.58$ & $18.67 \pm 0.58$ & $6.33 \pm 1.15$ & $5.33 \pm 1.53$ & $6.67 \pm 1.15$ & $5.67 \pm 1.15$ \\
\hline Staphylococcus haemoliticus 24.2 & $10.33 \pm 0.58$ & $10.67 \pm 0.58$ & $19.33 \pm 1.15$ & $8.67 \pm 1.15$ & $5.67 \pm 1.15$ & $6.67 \pm 0.58$ & $4.67 \pm 1.15$ \\
\hline Staphylococcus haemoliticus 24.5 & $12.17 \pm 0.29$ & $9.67 \pm 0.58$ & $18.67 \pm 0.58$ & $7.67 \pm 0.58$ & $6.67 \pm 0.58$ & $6.00 \pm 1.00$ & $5.33 \pm 0.58$ \\
\hline Staphylococcus haemoliticus 24.6 & $10.67 \pm 0.58$ & $8.33 \pm 0.58$ & $17.67 \pm 0.58$ & $8.33 \pm 1.15$ & $5.67 \pm 0.58$ & $5.67 \pm 0.58$ & $4.33 \pm 0.58$ \\
\hline Staphylococcus haemoliticus 24.7 & $12.00 \pm 2.00$ & $8.33 \pm 0.58$ & $19.33 \pm 1.15$ & $8.00 \pm 1.00$ & $6.33 \pm 1.15$ & $4.67 \pm 0.58$ & $4.67 \pm 0.58$ \\
\hline Staphylococcus haemoliticus 24.8 & $10.33 \pm 0.58$ & $8.33 \pm 0.58$ & $18.33 \pm 0.58$ & $7.67 \pm 0.58$ & $6.33 \pm 0.58$ & $5.33 \pm 0.58$ & $5.83 \pm 0.76$ \\
\hline Staphylococcus haemoliticus & $11.33 \pm 0.58$ & $12.67 \pm 0.58$ & $20.33 \pm 0.58$ & $8.33 \pm 0.58$ & $7.67 \pm 0.58$ & $6.33 \pm 0.58$ & $4.67 \pm 0.58$ \\
\hline Staphylococus hominis 3.1 & $11.33 \pm 0.58$ & $10.33 \pm 0.58$ & $21.67 \pm 0.58$ & $10.67 \pm 0.58$ & $18.33 \pm 0.58$ & $8.00 \pm 1.73$ & $3.33 \pm 1.15$ \\
\hline Staphylococus hominis 3.2 & $9.33 \pm 0.58$ & $11.33 \pm 1.15$ & $22.00 \pm 1.73$ & $9.33 \pm 0.58$ & $19.67 \pm 0.58$ & $8.33 \pm 0.58$ & $3.67 \pm 0.58$ \\
\hline Staphylococus hominis 3.3 & $7.67 \pm 0.58$ & $10.00 \pm 1.73$ & $21.33 \pm 0.58$ & $8.33 \pm 0.58$ & $19.33 \pm 0.58$ & $8.00 \pm 1.73$ & $4.33 \pm 0.58$ \\
\hline Staphylococus hominis 3.4 & $11.67 \pm 0.58$ & & & & & $7.33 \pm 1.15$ & \\
\hline Staphylococus hominis 3.5 & $9.67 \pm 0.58$ & $9.67 \pm 0.58$ & $21.67 \pm 0.58$ & & & & \\
\hline Staphylococus hominis 3.7 & $10.33 \pm 1.15$ & $10.33 \pm 1.15$ & $18.67 \pm 0.58$ & $8.67 \pm 0.58$ & $21.67 \pm 0.58$ & $8.00 \pm 1.00$ & $4.33 \pm 0.58$ \\
\hline Staphylococus hominis 14.4 & $10.67 \pm 1.15$ & $9.33 \pm 1.15$ & $20.33 \pm 0.58$ & $7.33 \pm 0.58$ & $18.67 \pm 1.15$ & $7.33 \pm 1.15$ & $3.67 \pm 0.58$ \\
\hline Staphylococus hominis 16.1 & $10.67 \pm 1.53$ & $8.67 \pm 0.58$ & $18.67 \pm 0.58$ & $7.67 \pm 1.15$ & $17.67 \pm 1.15$ & $7.33 \pm 0.58$ & $3.67 \pm 1.15$ \\
\hline Staphylococus hominis 16.4 & $9.33 \pm 0.58$ & $8.33 \pm 0.58$ & $20.33 \pm 0.58$ & $7.33 \pm 1.15$ & $19.67 \pm 0.58$ & $9.33 \pm 0.58$ & $3.67 \pm 0.58$ \\
\hline Staphylococus hominis 17.4 & $11.33 \pm 1.15$ & $9.00 \pm 1.00$ & $21.67 \pm 0.58$ & $8.33 \pm 0.58$ & $19.33 \pm 0.58$ & $7.67 \pm 0.58$ & $5.33 \pm 0.58$ \\
\hline Staphylococus hominis 18.1 & $12.67 \pm 1.15$ & $10.67 \pm 0.58$ & $20.67 \pm 0.58$ & $8.67 \pm 0.58$ & $18.33 \pm 0.58$ & $8.67 \pm 0.58$ & $5.67 \pm 0.58$ \\
\hline Staphylococus hominis 18.8 & $9.00 \pm 1.00$ & $11.67 \pm 0.58$ & $21.67 \pm 0.58$ & $8.67 \pm 1.15$ & $17.33 \pm 0.58$ & $7.67 \pm 0.58$ & $4.33 \pm 0.58$ \\
\hline Staphylococus hominis 21.1 & $11.33 \pm 0.58$ & $11.67 \pm 0.58$ & $20.50 \pm 0.50$ & $8.55 \pm 0.50$ & $18.33 \pm 0.58$ & $8.33 \pm 0.58$ & $4.67 \pm 0.58$ \\
\hline Staphylococus hominis 21.2 & $11.00 \pm 1.00$ & $11.00 \pm 1.00$ & $21.33 \pm 0.58$ & $7.67 \pm 0.58$ & $19.33 \pm 1.15$ & $8.67 \pm 1.15$ & $4.00 \pm 0.00$ \\
\hline Staphylococus hominis 27.4 & $10.67 \pm 1.15$ & $9.33 \pm 0.58$ & $22.33 \pm 0.58$ & $7.67 \pm 0.58$ & $19.33 \pm 1.53$ & $7.67 \pm 0.58$ & $4.33 \pm 1.15$ \\
\hline
\end{tabular}

Note: 1-Amyris balsamifera L., 2-Boswelia carterii Birdw., 3-Canarium luzonicum (Blume) A. Gray, 4-Cinnamomum camphora (L.) J. Presl., 5-Cinnamomum camphora var. linaloolifera Y. Fuita, 6-Citrus $x$ aurantium L., 7-Gaultheria procumbens L. 
Table 4. Antimicrobial activity of EOs with disc diffusion method in $\mathrm{mm}$.

\begin{tabular}{|c|c|c|c|c|c|c|c|}
\hline Isolate/EOs & 8. & 9. & 10. & 11. & 12. & 13. & 14. \\
\hline Stapyloccocus aureus 18.3 & $23.33 \pm 0.58$ & $4.67 \pm 0.58$ & $14.67 \pm 0.58$ & $9.00 \pm 1.00$ & $10.67 \pm 1.15$ & $5.33 \pm 0.58$ & $11.33 \pm 0.58$ \\
\hline Staphyloccous capitis 3.6 & $25.33 \pm 0.58$ & $3.67 \pm 1.15$ & $8.67 \pm 0.58$ & $8.33 \pm 0.58$ & $12.67 \pm 1.15$ & $7.33 \pm 0.58$ & $12.67 \pm 0.58$ \\
\hline Staphylococcus epidermidis 2.2 & $14.66 \pm 0.58$ & $4.33 \pm 0.58$ & $6.67 \pm 0.58$ & $7.33 \pm 0.58$ & $8.33 \pm 0.58$ & $4.33 \pm 0.58$ & $6.67 \pm 1.15$ \\
\hline Staphylococcus epidermidis 2.4 & $12.67 \pm 0.58$ & $4.00 \pm 1.00$ & $5.67 \pm 0.58$ & $6.33 \pm 0.58$ & $8.67 \pm 1.15$ & $4.33 \pm 0.58$ & $6.67 \pm 1.15$ \\
\hline Staphylococcus epidermidis 16.2 & $14.33 \pm 0.58$ & $3.67 \pm 0.58$ & $6.33 \pm 1.15$ & $6.00 \pm 1.00$ & $9.00 \pm 1.00$ & $5.00 \pm 1.00$ & $6.67 \pm 0.58$ \\
\hline Staphylococcus epidermidis 18.4 & $14.50 \pm 0.87$ & $4.67 \pm 0.58$ & $7.33 \pm 0.58$ & $5.67 \pm 1.15$ & $9.67 \pm 1.15$ & $5.33 \pm 0.58$ & $7.00 \pm 1.73$ \\
\hline Staphylococcus epidermidis 19.5 & $11.67 \pm 0.58$ & $3.67 \pm 0.58$ & $6.33 \pm 0.58$ & $5.67 \pm 0.58$ & $8.33 \pm 0.58$ & $4.33 \pm 1.15$ & $7.67 \pm 0.58$ \\
\hline Staphylococcus epidermidis 24.2 & $12.67 \pm 1.53$ & $3.33 \pm 0.58$ & $7.33 \pm 0.58$ & $6.67 \pm 0.58$ & $9.33 \pm 0.58$ & $5.33 \pm 0.58$ & $8.33 \pm 0.58$ \\
\hline Staphylococcus epidermidis 27.3 & $11.33 \pm 1.15$ & $4.67 \pm 0.58$ & $5.67 \pm 0.58$ & $5.33 \pm 0.58$ & $8.67 \pm 0.58$ & $4.67 \pm 0.58$ & $8.33 \pm 0.58$ \\
\hline Staphylococcus haemoliticus 2.3 & $12.33 \pm 0.58$ & $4.67 \pm 1.15$ & $5.33 \pm 0.58$ & $10.67 \pm 1.15$ & $10.33 \pm 0.58$ & $5.33 \pm 0.58$ & $8.67 \pm 0.58$ \\
\hline Staphylococcus haemoliticus 14.5 & $9.33 \pm 0.58$ & $3.67 \pm 1.15$ & $5.33 \pm 0.58$ & $10.33 \pm 0.58$ & $9.00 \pm 1.00$ & $4.00 \pm 1.00$ & $7.33 \pm 0.58$ \\
\hline Staphylococcus haemoliticus 14.6 & $9.33 \pm 1.15$ & $3.33 \pm 0.58$ & $6.33 \pm 0.58$ & $11.00 \pm 1.00$ & $8.33 \pm 0.58$ & $4.67 \pm 0.58$ & $7.00 \pm 1.73$ \\
\hline Staphylococcus haemoliticus 14.7 & $9.67 \pm 1.15$ & $4.67 \pm 1.15$ & $5.67 \pm 1.15$ & $11.33 \pm 1.15$ & $9.33 \pm 1.15$ & $5.00 \pm 1.00$ & $7.33 \pm 0.58$ \\
\hline Staphylococcus haemoliticus 14.8 & $9.33 \pm 1.53$ & $4.67 \pm 0.58$ & $5.67 \pm 0.58$ & $10.33 \pm 2.08$ & $9.67 \pm 1.15$ & $4.67 \pm 0.58$ & $6.33 \pm 0.58$ \\
\hline Staphylococcus haemoliticus 17.1 & $10.67 \pm 1.15$ & $3.67 \pm 0.58$ & $5.67 \pm 1$ & $12.33 \pm 0.58$ & $8.33 \pm 1.53$ & $4.67 \pm 1.15$ & $7.67 \pm 1.53$ \\
\hline Staphylococcus haemoliticus 17.2 & $10.00 \pm 1.73$ & $3.67 \pm 0.58$ & $5.33 \pm 0.58$ & $10.67 \pm 0.58$ & $10.00 \pm 2.00$ & $5.00 \pm 0.00$ & $6.33 \pm 0.58$ \\
\hline Staphylococcus haemoliticus 17.3 & $10.33 \pm 1.53$ & $4.33 \pm 0.58$ & $5.33 \pm 1.15$ & $11.67 \pm 0.58$ & $9.67 \pm 1.53$ & $5.50 \pm 0.50$ & $8.33 \pm 0.58$ \\
\hline Staphylococcus haemoliticus 17.5 & $8.00 \pm 1.00$ & $4.67 \pm 0.58$ & $6.33 \pm 0.58$ & $12.67 \pm 1.15$ & $8.33 \pm 0.58$ & $4.67 \pm 0.58$ & $8.33 \pm 1.53$ \\
\hline Staphylococcus haemoliticus 17.6 & & & & & $9.00 \pm 1$ & & \\
\hline Staphylococcus haemoliticus 19.7 & $6.33 \pm 1.15$ & $3.33 \pm 1.15$ & $6.33 \pm 0.58$ & $9.33 \pm 1.15$ & $7.67 \pm 0.58$ & $5.67 \pm 1.15$ & $7.67 \pm 0.58$ \\
\hline Staphylococcus haemoliticus 19.8 & $7.33 \pm 0.58$ & $4.33 \pm 1.15$ & $5.67 \pm 0.58$ & $10.67 \pm 0.58$ & $8.33 \pm 0.58$ & $5.33 \pm 0.58$ & $6.67 \pm 1.15$ \\
\hline Staphylococcus haemoliticus 20.2 & $6.67 \pm 1.15$ & $3.67 \pm 1.15$ & $6.33 \pm 1.15$ & $10.33 \pm 2.08$ & $9.33 \pm 1$ & $3.67 \pm 0.58$ & $6.67 \pm 0.58$ \\
\hline Staphylococcus haemoliticus 20.4 & $8.33 \pm 1.15$ & $4.00 \pm 0.00$ & $6.00 \pm 1.73$ & $10.67 \pm 1.15$ & $10.67 \pm 1$ & $6.00 \pm 1.00$ & $5.33 \pm 0.58$ \\
\hline Staphylococcus haemoliticus 20.5 & $8.67 \pm 0.58$ & $3.67 \pm 1.15$ & $5.33 \pm 0.58$ & $12.33 \pm 0.58$ & $10.67 \pm 0.58$ & $4.67 \pm 0.58$ & $5.67 \pm 0.58$ \\
\hline Staphylococcus haemoliticus 21.5 & $8.33 \pm 0.58$ & $3.83 \pm 0.76$ & $6.67 \pm 1.53$ & $11.67 \pm 0.58$ & $10.67 \pm 1.15$ & $5.33 \pm 1.15$ & $4.66 \pm 1.15$ \\
\hline Staphylococcus haemoliticus 21.6 & $9.33 \pm 0.58$ & $4.33 \pm 0.58$ & $8.67 \pm 0.58$ & $11.33 \pm 1.15$ & $9.00 \pm 1.00$ & $4.67 \pm 0.58$ & $6.33 \pm 1.15$ \\
\hline Staphylococcus haemoliticus 21.7 & $8.00 \pm 1.73$ & $4.33 \pm 0.58$ & $8.67 \pm 1.15$ & $11.33 \pm 0.58$ & $10.67 \pm 1.15$ & $5.33 \pm 0.58$ & $6.67 \pm 1.15$ \\
\hline Staphylococcus haemoliticus 22.5 & $7.33 \pm 0.58$ & $3.67 \pm 0.58$ & $8.33 \pm 1.15$ & $10.67 \pm 1.15$ & $9.00 \pm 1.00$ & $5.00 \pm 1.73$ & $6.33 \pm 0.58$ \\
\hline Staphylococcus haemoliticus 24.1 & $10.00 \pm 1.00$ & $4.33 \pm 0.58$ & $8.00 \pm 1.00$ & $10.67 \pm 0.58$ & $9.00 \pm 1.73$ & $5.00 \pm 0.87$ & $7.00 \pm 1.73$ \\
\hline Staphylococcus haemoliticus 24.2 & & & & & & & \\
\hline Staphylococcus haemoliticus 24.5 & $9.67 \pm 1.15$ & $4.67 \pm 1.15$ & $8.33 \pm 1.15$ & $12.67 \pm 1.15$ & $10.67 \pm 1.15$ & $5.67 \pm 1.15$ & $7.33 \pm 0.58$ \\
\hline Staphylococcus haemoliticus 24.6 & $7.67 \pm 0.58$ & $4.67 \pm 0.58$ & $9.67 \pm 0.58$ & $10.67 \pm 0.58$ & $9.33 \pm 1.15$ & $5.33 \pm 0.58$ & $6.67 \pm 1.15$ \\
\hline Staphylococcus haemoliticus 24.7 & $8.00 \pm 1.00$ & $5.67 \pm 0.58$ & $8.33 \pm 0.58$ & $12.33 \pm 0.58$ & $9.33 \pm 1.53$ & $4.33 \pm 0.58$ & $6.67 \pm 1.53$ \\
\hline Staphylococcus haemoliticus 24.8 & & & & & & & \\
\hline Staphylococcus haemoliticus & $6.67 \pm 0.58$ & $4.67 \pm 0.58$ & $8.67 \pm 1.15$ & $12.67 \pm 1.15$ & $9.67 \pm 0.58$ & $5.33 \pm 0.58$ & $5.33 \pm 0.58$ \\
\hline Staphylococus hominis 3.1 & $21.33 \pm 1.15$ & $6.67 \pm 0.58$ & $15.33 \pm 0.58$ & $8.67 \pm 1.15$ & $8.33 \pm 0.58$ & $7.67 \pm 0.58$ & $10.67 \pm 1.15$ \\
\hline Staphylococus hominis 3.2 & $20.67 \pm 0.58$ & $5.67 \pm 0.58$ & $14.33 \pm 0.58$ & $8.33 \pm 0.58$ & $7.33 \pm 0.58$ & $6.67 \pm 0.58$ & $11.33 \pm 0.58$ \\
\hline Staphylococus hominis 3.3 & $19.33 \pm 0.58$ & $6.33 \pm 1.15$ & $15.33 \pm 0.58$ & $10.33 \pm 0.58$ & $8.33 \pm 0.58$ & $7.67 \pm 0.58$ & $11.67 \pm 0.58$ \\
\hline Staphylococus hominis 3.4 & $21.00 \pm 1.73$ & $6.67 \pm 1.15$ & $14.33 \pm 0.58$ & $10.67 \pm 1.15$ & $7.67 \pm 0.58$ & $8.67 \pm 0.58$ & $11.67 \pm 1.53$ \\
\hline Staphylococus hominis 3.5 & $21.67 \pm 1.15$ & $5.67 \pm 0.58$ & $13.33 \pm 0.58$ & $9.67 \pm 0.58$ & $7.67 \pm 1.15$ & $7.67 \pm 0.58$ & $11.67 \pm 0.58$ \\
\hline Staphylococus hominis 3.7 & $20.67 \pm 1.15$ & $5.33 \pm 1.53$ & $14.33 \pm 0.58$ & $12.33 \pm 0.58$ & $8.33 \pm 0.58$ & $7.67 \pm 0.58$ & $12.33 \pm 0.58$ \\
\hline Staphylococus hominis 14.4 & $21.67 \pm 0.58$ & $4.67 \pm 0.58$ & $13.67 \pm 1.53$ & $10.33 \pm 0.58$ & $7.67 \pm 0.58$ & $8.33 \pm 0.58$ & $12.67 \pm 1.15$ \\
\hline Staphylococus hominis 16.1 & $22.67 \pm 1.15$ & $5.67 \pm 0.58$ & $11.33 \pm 1.15$ & $12.67 \pm 1.15$ & $6.67 \pm 1.15$ & $6.67 \pm 1.15$ & $11.33 \pm 1.15$ \\
\hline Staphylococus hominis 16.4 & $20.33 \pm 1.15$ & $6.67 \pm 0.58$ & $14.33 \pm 0.58$ & $10.33 \pm 1.15$ & $6.33 \pm 0.58$ & $7.33 \pm 0.58$ & $10.33 \pm 0.58$ \\
\hline Staphylococus hominis 17.4 & $22.33 \pm 0.58$ & $6.33 \pm 0.58$ & $12.67 \pm 1.15$ & $11.33 \pm 1.15$ & $5.33 \pm 1.15$ & $8.67 \pm 0.58$ & $10.67 \pm 0.58$ \\
\hline Staphylococus hominis 18.1 & $22.67 \pm 0.58$ & $6.00 \pm 1.00$ & $11.33 \pm 1.15$ & $9.33 \pm 0.58$ & $6.33 \pm 1.15$ & $7.33 \pm 0.58$ & $9.33 \pm 0.58$ \\
\hline Staphylococus hominis 18.8 & $19.33 \pm 0.58$ & $7.00 \pm 1.73$ & $11.67 \pm 1.15$ & $11.67 \pm 1.53$ & $5.67 \pm 1.15$ & $8.67 \pm 0.58$ & $11.33 \pm 0.58$ \\
\hline Staphylococus hominis 21.1 & $18.67 \pm 0.58$ & $6.33 \pm 0.58$ & $12.67 \pm 0.50$ & $13.67 \pm 1.53$ & $7.33 \pm 1.15$ & $6.33 \pm 1.53$ & $11.67 \pm 0.58$ \\
\hline Staphylococus hominis 21.2 & $18.33 \pm 0.58$ & $7.67 \pm 0.58$ & $12.00 \pm 1.73$ & $11.33 \pm 1.53$ & $6.33 \pm 0.58$ & $7.00 \pm 1.73$ & $12.33 \pm 0.58$ \\
\hline Staphylococus hominis 27.4 & $19.00 \pm 1.73$ & $7.67 \pm 1.15$ & $11.67 \pm 0.58$ & $12.67 \pm 0.58$ & $7.33 \pm 0.58$ & $8.33 \pm 0.58$ & $12.33 \pm 0.58$ \\
\hline
\end{tabular}

Note: 8-Litsea cubeba (Lour.) Pers., 9-Melaleuca leucadendron L., 10-Melaleuca ericifolia Smith., 11-Pogostemon cabli (Blanco) Benth., 12-Citrus limon (L.) Osbeck, 13-Santalum album L., 14-Vetiveria zizanoides (L.) Roberty.

Table 5. Antimicrobial activity of EO detected with minimal inhibitory concentration in $\mu \mathrm{L} / \mathrm{mL}$.

\begin{tabular}{lccccccc}
\hline \multicolumn{1}{c}{ Microorganism/EOs } & $\mathbf{1 .}$ & $\mathbf{2 .}$ & $\mathbf{3 .}$ & $\mathbf{4 .}$ & $\mathbf{5 .}$ & $\mathbf{6 .}$ & $\mathbf{7 .}$ \\
\hline Stapyloccocus aureus 18.3 & 1.56 & 1.56 & 0.39 & 25.00 & 0.39 & 3.12 & 12.50 \\
Staphyloccous capitis 3.6 & 1.56 & 3.12 & 0.78 & 25.00 & 1.56 & 12.50 & 12.50 \\
Staphylococcus epidermidis 2.2 & 3.12 & 1.56 & 0.78 & 12.50 & 3.12 & 3.12 & 25.00 \\
Staphylococcus epidermidis 2.4 & 3.12 & 1.56 & 0.78 & 25.00 & 3.12 & 3.12 & 25.00 \\
Staphylococcus epidermidis 16.2 & 3.12 & 1.56 & 0.39 & 25.00 & 3.12 & 3.12 & 25.00 \\
Staphylococcus epidermidis 18.4 & 3.12 & 1.56 & 1.56 & 25.00 & 3.12 & 3.12 & 25.00 \\
Staphylococcus epidermidis 19.5 & 1.56 & 1.56 & 0.78 & 25.00 & 3.12 & 3.12 & 25.00 \\
Staphylococcus epidermidis 24.2 & 3.12 & 1.56 & 1.56 & 25.00 & 3.12 & 3.12 & 25.00 \\
Staphylococcus epidermidis 27.3 & 3.12 & 1.56 & 0.78 & 25.00 & 3.12 & 3.12 & 25.00 \\
Staphylococcus haemoliticus 2.3 & 6.25 & 6.25 & 0.78 & 25.00 & 12.50 & 6.25 & 25.00 \\
Staphylococcus haemoliticus 14.5 & 6.25 & 6.25 & 1.56 & 25.00 & 12.50 & 12.50 & 25.00 \\
\hline
\end{tabular}


Table 5. Cont.

\begin{tabular}{|c|c|c|c|c|c|c|c|}
\hline Microorganism/EOs & 1. & 2. & 3. & 4. & 5. & 6. & 7. \\
\hline Staphylococcus haemoliticus 14.6 & 3.12 & 6.25 & 1.56 & 25.00 & 12.50 & 12.50 & 25.00 \\
\hline Staphylococcus haemoliticus 14.7 & 12.50 & 6.25 & 0.78 & 3.12 & 12.50 & 12.50 & 25.00 \\
\hline Staphylococcus haemoliticus 14.8 & 3.12 & 6.25 & 1.56 & 3.12 & 12.50 & 12.50 & 25.00 \\
\hline Staphylococcus haemoliticus 17.1 & 3.12 & 3.12 & 1.56 & 25.00 & 12.50 & 12.50 & 25.00 \\
\hline Staphylococcus haemoliticus 17.2 & 3.12 & 3.12 & 1.56 & 25.00 & 12.50 & 12.50 & 25.00 \\
\hline Staphylococcus haemoliticus 17.3 & 3.12 & 6.25 & 1.56 & 25.00 & 12.50 & 12.50 & 25.00 \\
\hline Staphylococcus haemoliticus 17.5 & 1.56 & 6.25 & 1.56 & 25.00 & 6.25 & 25.00 & 25.00 \\
\hline Staphylococcus haemoliticus 17.6 & 3.12 & 6.25 & 0.78 & 25.00 & 6.25 & 25.00 & 25.00 \\
\hline Staphylococcus haemoliticus 19.7 & 1.56 & 6.25 & 1.56 & 25.00 & 6.25 & 25.00 & 25.00 \\
\hline Staphylococcus haemoliticus 19.8 & 1.56 & 3.12 & 1.56 & 25.00 & 6.25 & 12.50 & 12.50 \\
\hline Staphylococcus haemoliticus 20.2 & 6.25 & 3.12 & 0.78 & 25.00 & 12.50 & 12.50 & 25.00 \\
\hline Staphylococcus haemoliticus 20.4 & 6.25 & 6.25 & 1.56 & 25.00 & 12.50 & 12.50 & 25.00 \\
\hline Staphylococcus haemoliticus 20.5 & 1.56 & 6.25 & 1.56 & 25.00 & 25.00 & 12.50 & 12.50 \\
\hline Staphylococcus haemoliticus 21.5 & 1.56 & 6.25 & 0.78 & 25.00 & 12.50 & 12.50 & 25.00 \\
\hline Staphylococcus haemoliticus 21.6 & 3.12 & 6.25 & 1.56 & 25.00 & 12.50 & 12.50 & 12.50 \\
\hline Staphylococcus haemoliticus 21.7 & 6.25 & 3.12 & 1.56 & 25.00 & 25.00 & 12.50 & 25.00 \\
\hline Staphylococcus haemoliticus 22.5 & 6.25 & 6.25 & 1.56 & 25.00 & 25.00 & 12.50 & 25.00 \\
\hline Staphylococcus haemoliticus 24.1 & 3.12 & 1.56 & 1.56 & 25.00 & 12.50 & 12.50 & 25.00 \\
\hline Staphylococcus haemoliticus 24.2 & 3.12 & 1.56 & 1.56 & 25.00 & 12.50 & 12.50 & 25.00 \\
\hline Staphylococcus haemoliticus 24.5 & 1.56 & 3.12 & 1.56 & 25.00 & 12.50 & 12.50 & 25.00 \\
\hline Staphylococcus haemoliticus 24.6 & 1.56 & 3.12 & 1.56 & 25.00 & 12.50 & 25.00 & 25.00 \\
\hline Staphylococcus haemoliticus 24.7 & 1.56 & 3.12 & 1.56 & 25.00 & 12.50 & 25.00 & 25.00 \\
\hline Staphylococcus haemoliticus 24.8 & 1.56 & 3.12 & 1.56 & 25.00 & 12.50 & 25.00 & 25.00 \\
\hline Staphylococcus haemoliticus & 1.56 & 1.56 & 0.78 & 25.00 & 12.50 & 12.50 & 25.00 \\
\hline Staphylococus hominis 3.1 & 1.56 & 1.56 & 0.78 & 12.50 & 3.12 & 12.50 & 25.00 \\
\hline Staphylococus hominis 3.2 & 3.12 & 1.56 & 0.78 & 12.50 & 3.12 & 12.50 & 25.00 \\
\hline Staphylococus hominis 3.3 & 6.25 & 1.56 & 0.78 & 12.50 & 3.12 & 12.50 & 25.00 \\
\hline Staphylococus hominis 3.4 & 3.12 & 3.12 & 0.78 & 12.50 & 3.12 & 12.50 & 25.00 \\
\hline Staphylococus hominis 3.5 & 3.12 & 3.12 & 0.78 & 12.50 & 1.56 & 12.50 & 25.00 \\
\hline Staphylococus hominis 3.7 & 3.12 & 1.56 & 1.56 & 12.50 & 0.78 & 12.50 & 25.00 \\
\hline Staphylococus hominis 14.4 & 3.12 & 3.12 & 0.78 & 12.50 & 1.56 & 12.50 & 25.00 \\
\hline Staphylococus hominis 16.1 & 3.12 & 3.12 & 1.56 & 6.25 & 1.56 & 12.50 & 25.00 \\
\hline Staphylococus hominis 16.4 & 3.12 & 3.12 & 0.78 & 12.50 & 3.12 & 12.50 & 25.00 \\
\hline Staphylococus hominis 17.4 & 1.56 & 3.12 & 0.78 & 25.00 & 3.12 & 12.50 & 25.00 \\
\hline Staphylococus hominis 18.1 & 1.56 & 3.12 & 0.78 & 25.00 & 3.12 & 12.50 & 25.00 \\
\hline Staphylococus hominis 18.8 & 3.12 & 3.12 & 0.78 & 12.50 & 1.56 & 12.50 & 25.00 \\
\hline Staphylococus hominis 21.1 & 3.12 & 3.12 & 0.78 & 12.50 & 1.56 & 12.50 & 25.00 \\
\hline Staphylococus hominis 21.2 & 3.12 & 3.12 & 0.78 & 25.00 & 1.56 & 12.50 & 25.00 \\
\hline Staphylococus hominis 27.4 & 3.12 & 6.25 & 0.39 & 25.00 & 3.12 & 12.50 & 25.00 \\
\hline
\end{tabular}

Note: 1-Amyris balsamifera L., 2-Boswelia carterii Birdw., 3-Canarium luzonicum (Blume) A. Gray, 4-Cinnamomum camphora (L.) J. Presl., 5-Cinnamomum camphora var. linaloolifera Y. Fuita, 6-Citrus $\mathrm{x}$ aurantium L., 7-Gaultheria procumbens L.

Table 6. Minimal inhibitory concentration of EOs in $\mu \mathrm{L} / \mathrm{mL}$.

\begin{tabular}{lccccccc}
\hline \multicolumn{1}{c}{ Name of bacteria/EOs } & $\mathbf{8 .}$ & $\mathbf{9 .}$ & $\mathbf{1 0 .}$ & $\mathbf{1 1 .}$ & $\mathbf{1 2 .}$ & $\mathbf{1 3 .}$ & $\mathbf{1 4 .}$ \\
\hline Stapyloccocus aureus 18.3 & 0.39 & 25.00 & 3.12 & 3.12 & 3.12 & 12.50 & 3.12 \\
Staphyloccous capitis 3.6 & 0.39 & 25.00 & 6.25 & 3.12 & 3.12 & 6.25 & 3.12 \\
Staphylococcus epidermidis 2.2 & 1.56 & 25.00 & 12.50 & 6.25 & 6.25 & 12.50 & 25.00 \\
Staphylococcus epidermidis 2.4 & 1.56 & 25.00 & 12.50 & 6.25 & 6.25 & 12.50 & 25.00 \\
Staphylococcus epidermidis 16.2 & 1.56 & 25.00 & 12.50 & 6.25 & 6.25 & 12.50 & 25.00 \\
Staphylococcus epidermidis 18.4 & 1.56 & 25.00 & 12.50 & 6.25 & 6.25 & 12.50 & 25.00 \\
Staphylococcus epidermidis 19.5 & 1.56 & 25.00 & 12.50 & 6.25 & 6.25 & 12.50 & 12.50 \\
Staphylococcus epidermidis 24.2 & 1.56 & 25.00 & 12.50 & 6.25 & 6.25 & 12.50 & 12.50 \\
Staphylococcus epidermidis 27.3 & 1.56 & 25.00 & 12.50 & 6.25 & 6.25 & 12.50 & 12.50 \\
Staphylococcus haemoliticus 2.3 & 1.56 & 25.00 & 12.50 & 3.12 & 3.12 & 12.50 & 12.50 \\
Staphylococcus haemoliticus 14.5 & 3.12 & 25.00 & 12.50 & 3.12 & 3.12 & 12.50 & 12.50 \\
Staphylococcus haemoliticus 14.6 & 3.12 & 25.00 & 12.50 & 3.12 & 3.12 & 12.50 & 12.50 \\
Staphylococcus haemoliticus 14.7 & 3.12 & 25.00 & 12.50 & 3.12 & 3.12 & 12.50 & 12.50 \\
Staphylococcus haemoliticus 14.8 & 3.12 & 25.00 & 12.50 & 3.12 & 3.12 & 12.50 & 12.50 \\
\hline
\end{tabular}


Table 6. Cont.

\begin{tabular}{|c|c|c|c|c|c|c|c|}
\hline Name of bacteria/EOs & 8. & 9. & 10. & 11. & 12. & 13. & 14. \\
\hline Staphylococcus haemoliticus 17.1 & 3.12 & 25.00 & 12.50 & 3.12 & 3.12 & 12.50 & 12.50 \\
\hline Staphylococcus haemoliticus 17.2 & 3.12 & 25.00 & 12.50 & 3.12 & 3.12 & 12.50 & 12.50 \\
\hline Staphylococcus haemoliticus 17.3 & 3.12 & 25.00 & 12.50 & 3.12 & 3.12 & 12.50 & 12.50 \\
\hline Staphylococcus haemoliticus 17.5 & 6.25 & 25.00 & 12.50 & 3.12 & 3.12 & 25.00 & 12.50 \\
\hline Staphylococcus haemoliticus 17.6 & 6.25 & 25.00 & 12.50 & 3.12 & 3.12 & 25.00 & 12.50 \\
\hline Staphylococcus haemoliticus 19.7 & 6.25 & 25.00 & 12.50 & 3.12 & 6.25 & 25.00 & 12.50 \\
\hline Staphylococcus haemoliticus 19.8 & 6.25 & 25.00 & 12.50 & 3.12 & 6.25 & 25.00 & 12.50 \\
\hline Staphylococcus haemoliticus 20.2 & 6.25 & 25.00 & 12.50 & 3.12 & 6.25 & 25.00 & 12.50 \\
\hline Staphylococcus haemoliticus 20.4 & 6.25 & 25.00 & 12.50 & 3.12 & 3.12 & 25.00 & 25.00 \\
\hline Staphylococcus haemoliticus 20.5 & 6.25 & 25.00 & 12.50 & 3.12 & 3.12 & 25.00 & 25.00 \\
\hline Staphylococcus haemoliticus 21.5 & 6.25 & 25.00 & 12.50 & 3.12 & 3.12 & 25.00 & 25.00 \\
\hline Staphylococcus haemoliticus 21.6 & 6.25 & 25.00 & 6.25 & 3.12 & 3.12 & 25.00 & 25.00 \\
\hline Staphylococcus haemoliticus 21.7 & 6.25 & 25.00 & 6.25 & 3.12 & 3.12 & 25.00 & 25.00 \\
\hline Staphylococcus haemoliticus 22.5 & 6.25 & 25.00 & 6.25 & 3.12 & 3.12 & 25.00 & 25.00 \\
\hline Staphylococcus haemoliticus 24.1 & 3.12 & 25.00 & 6.25 & 3.12 & 3.12 & 25.00 & 25.00 \\
\hline Staphylococcus haemoliticus 24.2 & 3.12 & 25.00 & 6.25 & 3.12 & 3.12 & 25.00 & 25.00 \\
\hline Staphylococcus haemoliticus 24.5 & 3.12 & 25.00 & 6.25 & 3.12 & 3.12 & 25.00 & 25.00 \\
\hline Staphylococcus haemoliticus 24.6 & 6.25 & 25.00 & 6.25 & 3.12 & 3.12 & 25.00 & 25.00 \\
\hline Staphylococcus haemoliticus 24.7 & 6.25 & 25.00 & 6.25 & 3.12 & 3.12 & 25.00 & 25.00 \\
\hline Staphylococcus haemoliticus 24.8 & 6.25 & 25.00 & 6.25 & 3.12 & 3.12 & 25.00 & 25.00 \\
\hline Staphylococcus haemoliticus & 6.25 & 25.00 & 6.25 & 3.12 & 3.12 & 25.00 & 25.00 \\
\hline Staphylococus hominis 3.1 & 0.78 & 12.50 & 3.12 & 6.25 & 3.12 & 6.25 & 3.12 \\
\hline Staphylococus hominis 3.2 & 0.78 & 12.50 & 3.12 & 6.25 & 6.25 & 6.25 & 3.12 \\
\hline Staphylococus hominis 3.3 & 0.78 & 12.50 & 3.12 & 3.12 & 3.12 & 6.25 & 3.12 \\
\hline Staphylococus hominis 3.4 & 0.78 & 12.50 & 3.12 & 3.12 & 3.12 & 6.25 & 3.12 \\
\hline Staphylococus hominis 3.5 & 0.78 & 25.00 & 3.12 & 3.12 & 3.12 & 6.25 & 3.12 \\
\hline Staphylococus hominis 3.7 & 0.78 & 25.00 & 3.12 & 3.12 & 3.12 & 6.25 & 3.12 \\
\hline Staphylococus hominis 14.4 & 0.78 & 25.00 & 3.12 & 3.12 & 3.12 & 6.25 & 3.12 \\
\hline Staphylococus hominis 16.1 & 0.78 & 25.00 & 3.12 & 3.12 & 3.12 & 6.25 & 3.12 \\
\hline Staphylococus hominis 16.4 & 0.78 & 12.50 & 3.12 & 3.12 & 3.12 & 6.25 & 3.12 \\
\hline Staphylococus hominis 17.4 & 0.78 & 12.50 & 3.12 & 3.12 & 3.12 & 6.25 & 3.12 \\
\hline Staphylococus hominis 18.1 & 0.78 & 12.50 & 3.12 & 6.25 & 3.12 & 6.25 & 3.12 \\
\hline Staphylococus hominis 18.8 & 0.78 & 12.50 & 3.12 & 3.12 & 3.12 & 6.25 & 3.12 \\
\hline Staphylococus hominis 21.1 & 0.78 & 12.50 & 3.12 & 3.12 & 6.25 & 6.25 & 3.12 \\
\hline Staphylococus hominis 21.2 & 0.78 & 12.50 & 3.12 & 3.12 & 6.25 & 6.25 & 3.12 \\
\hline Staphylococus hominis 27.4 & 0.78 & 12.50 & 3.12 & 3.12 & 6.25 & 6.25 & 3.12 \\
\hline
\end{tabular}

The best antimicrobial activity of A. balsamifera L. was found against S. aureus $(16.50 \pm 1.32 \mathrm{~mm})$. B. carterrii Birdw. revealed the best antimicrobial effect against $S$. epidermidis $(13.33 \pm 1.15 \mathrm{~mm})$. C. luzonicum (Blume) A. Gray showed the best antimicrobial activity against S. capitis $(24.67 \pm 0.58 \mathrm{~mm})$, and C. camphora (L.) J. Presl. was found to be most effective against S. hominis (10.67 $\pm 0.58 \mathrm{~mm}$ ). The best antimicrobial activity of $C$. camphora var. linaloolifera $Y$. Fuita was recorded against $S$. aureus $(24.67 \pm 0.58 \mathrm{~mm})$, and $C$. $x$ aurantium $\mathrm{L}$. exhibited the highest antimicrobial properties against S. epidermidis $(17.33 \pm 0.58 \mathrm{~mm})$. The $\mathrm{EO}$ of G. procumbens L. was most effective against S. capitis $(8.33 \pm 0.58 \mathrm{~mm})$.

The best antimicrobial activity of $L$. cubeba (Lour.) Pers was found against $S$. capitis ( $25.33 \pm 0.58 \mathrm{~mm})$. M. leucadendron $\mathrm{L}$. showed the best antimicrobial effect against $S$. hominis $(7.67 \pm 0.58$ and $7.67 \pm 1.15 \mathrm{~mm}$, respectively). M. ericifolia Smith. was highly effective against $S$. hominis $(15.33 \pm 0.58 \mathrm{~mm})$, while $P$. cabli (Blanco) Benth. exhibited the highest antimicrobial potential against S. haemoliticus and S. hominis $(12.67 \mathrm{~mm})$. The best antimicrobial activity of C. limon (L.) Osbeck was found against S. capitis $(12.67 \pm 1.15 \mathrm{~mm})$, and S. album L. was highly efficient against S. hominis $(8.67 \pm 0.58 \mathrm{~mm})$. The most effective antimicrobial activity of $V$. zizanoides (L.) Roberty EO was recorded against $S$. capitis and S. hominis (12.67 mm). 
For the analysed EOs, significant differences in their activity were observed against Staphylococcus spp. (Table 7). The most pronounced activity was recorded for C.luzonicum (Blume) A. Gray, A. Balsamifera L., C. camphora var. linaloolifera, and P. cabli (Blanco) Benth. EOs.

Table 7. Differences between the analysed essential oils in their activity against Staphylococcus spp.

\begin{tabular}{|c|c|}
\hline Essential Oil & Average/Standard Deviation/Statistical Difference \\
\hline A. balsamifera $\mathrm{L}$. & $10.82 \pm 2.09^{\mathrm{a}}$ \\
\hline B. carterii Birdw. & $9.95 \pm 1.64^{\mathrm{b}, \mathrm{a}}$ \\
\hline C. luzonicum (Blume) A. Gray & $19.84 \pm 1.94^{\mathrm{c}, \mathrm{a}, \mathrm{b}}$ \\
\hline C. camphora (L.) J. Presl. & $7.18 \pm 1.60^{\mathrm{d}, \mathrm{a}, \mathrm{b}, \mathrm{c}}$ \\
\hline C. camphora var. linaloolifera Y. Fuita & $10.65 \pm 6.30^{\mathrm{e}, \mathrm{c}, \mathrm{d}}$ \\
\hline C. $x$ aurantium $\mathrm{L}$. & $8.84 \pm 3.33^{\mathrm{f}, \mathrm{a}, \mathrm{b}, \mathrm{c}, \mathrm{d}, \mathrm{e}}$ \\
\hline G. procumbens L. & $5.07 \pm 1.13 \mathrm{~g}, \mathrm{a}, \mathrm{b}, \mathrm{c}, \mathrm{d}, \mathrm{e}, \mathrm{f}$ \\
\hline L. cubeba (Lour.) Pers. & $13.54 \pm 5.90^{\mathrm{h}, \mathrm{a}, \mathrm{b}, \mathrm{c}, \mathrm{d}, \mathrm{e}, \mathrm{g}}$ \\
\hline M. leucadendron L. & $4.82 \pm 1.34^{\mathrm{i}, \mathrm{a}, \mathrm{b}, \mathrm{c}, \mathrm{d}, \mathrm{e}, \mathrm{f}, \mathrm{h}}$ \\
\hline M. ericifolia Smith. & $8.93 \pm 3.40 \mathrm{j}, \mathrm{a}, \mathrm{b}, \mathrm{c}, \mathrm{d}, \mathrm{e}, \mathrm{g}, \mathrm{h}, \mathrm{i}$ \\
\hline P. cabli (Blanco) Benth. & $10.29 \pm 2.20^{\mathrm{k}, \mathrm{c}, \mathrm{d}, \mathrm{g}, \mathrm{h}, \mathrm{i}, \mathrm{j}}$ \\
\hline C. limon (L.) Osbeck & $8.71 \pm 1.66^{1, a, b, c, d, e, g, h, i, k}$ \\
\hline S. album L. & $5.81 \pm 1.53 \mathrm{~m}, \mathrm{a}, \mathrm{b}, \mathrm{c}, \mathrm{d}, \mathrm{e}, \mathrm{g}, \mathrm{h}, \mathrm{i}, \mathrm{j}, \mathrm{k}, \mathrm{l}$ \\
\hline V. zizanoides (L.) Roberty & $8.47 \pm 2.49^{n, a, b, c, d}, \mathrm{e}, \mathrm{g}, \mathrm{h}, \mathrm{i}, \mathrm{k}, \mathrm{m}$ \\
\hline
\end{tabular}

Note: Individual letters (a-n) in upper case indicate the statistical difference. $p \leq 0.05$.

No significant differences were found against A. balsamifera L. vs. P. cabli (Blanco) Benth.; G. procumbens L. vs. M. leucadendron L.; B. carterii Birdw. vs. P. cabli (Blanco) Benth.; M. ericifolia Smith. vs. V. zizanoides (L.) Roberty; B. carterii Birdw. vs. C. camphora var. linaloolifera Y. Fuita; C. $x$ aurantium L. vs. V. zizanoides (L.) Roberty; C. limon (L.) Osbeck vs. V. zizanoides (L.) Roberty; M. ericifolia Smith. vs. C. limon (L.) Osbeck; C. camphora var. linaloolifera Y. Fuita vs. P. cabli (Blanco) Benth.; C. $x$ aurantium L. vs. C. limon (L.) Osbeck; A. balsamifera L. vs. C. camphora var. linaloolifera Y. Fuita; and C. $x$ aurantium L. vs. M. ericifolia Smith (Figure 2).

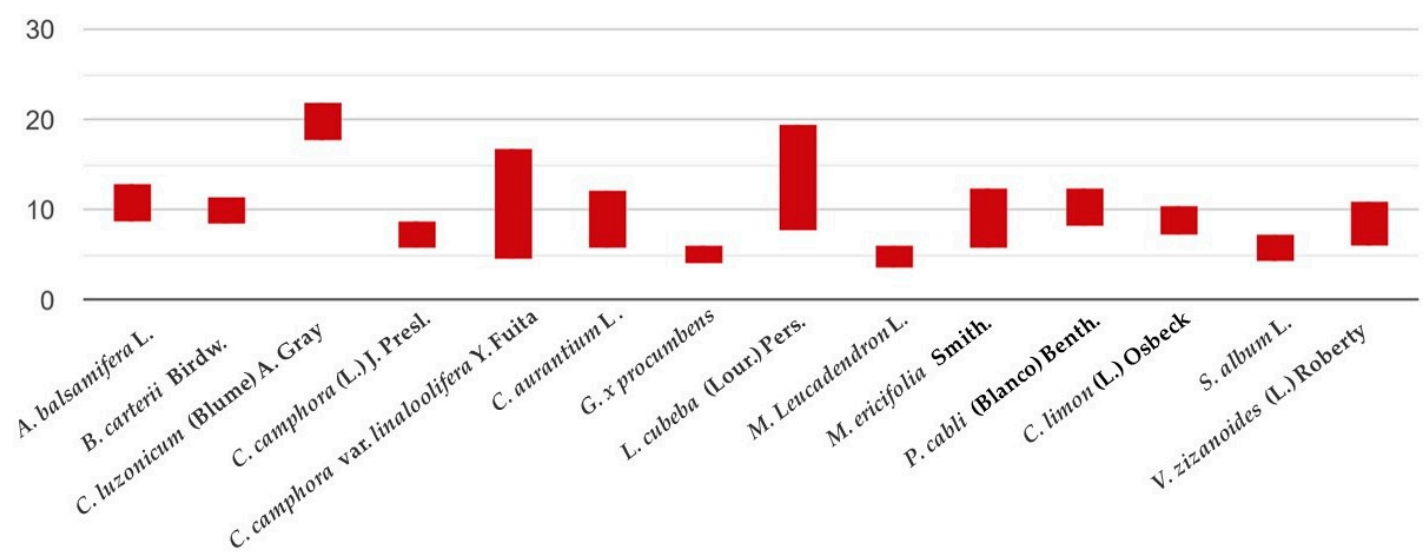

Figure 2. Mean (mm) and standard deviation for analysed essential oils in their activity against Staphylococcus spp.

In this study, the EO of $A$. balsamifera L. showed the best antimicrobial activity with the disc diffusion test against $S$. aureus with an inhibition zone of $16.50 \mathrm{~mm}$. Minimum inhibitory concentration (MIC) values obtained with the broth microdilution method were $1.59 \mu \mathrm{L} / \mathrm{mL}$ against S. aureus, S. capitis, one strain of $S$. epidermidis, 10 strains of $S$. haemoliticus, and three strains of S. hominis. A. balsamifera was reported to possess antimicrobial activity against gram-positive and gram-negative bacteria, including Staphylococcus aureus, Salmonella paratyphi, Escherichia coli, Klebsiella pneumoniae, and microscopic fungi [46]. 
B. carterii Birdw. EO was found to be the most effective against one strain of S. epidermidis $(13.33 \mathrm{~mm})$ tested with the disc diffusion method. With the microdilution method, MIC $=1.59 \mu \mathrm{L} / \mathrm{mL}$ was found against $S$. aureus, all strains of $S$. epidermidis, three strains of $S$. haemoliticus, and three strains of $S$. hominis. The antimicrobial activity of EOs of B. carteri, B. neglecta, B. sacra, B. thurifera, and B. frereana varied from moderate to poor against S. aureus (ATCC 12600) [47].

The EO of C. luzonicum (Blume) A. Gray exhibited the best antimicrobial activity against $S$. capitis $(24.67 \mathrm{~mm}$ ) with the disc diffusion method. Using the broth microdilitution method, MIC $=0.39 \mu \mathrm{L} / \mathrm{mL}$ was recorded against S. aureus, one strain of S. epidermidis, and one strain of S. hominis. C. luzonicum was reported to show antifungal activity without expressing toxicity or other negative side effects [48].

C. camphora (L.) J. Presl. EO revealed the best antimicrobial activity against $S$. homins with an inhibition zone of $10.67 \mathrm{~mm}$ with the disc diffusion test and MIC $=3.12 \mu \mathrm{L} / \mathrm{mL}$ against two strains of S. haemoliticus. C. camphora var. linaloolifera Y. Fuita showed the best antimicrobial activity against S. aureus, with an inhibition zone of $24.67 \mathrm{~mm}$ with the disc diffusion method and MIC $=0.39 \mu \mathrm{L} / \mathrm{mL}$ against $S$. aureus. The EO of $C$. camphora was found to possess antifungal activity against $A$. niger (MIC $=20 \mu \mathrm{g} / \mathrm{mL}$ ) and exhibited an inhibitory effect against B. cereus and S. aureus [49]. Previously identified antimicrobial properties of the EOs of $C$. camphora were in agreement with our results [50-54].

The EO of C. $x$ aurantium was the most active against one strain of $S$. epidermidis with the disc diffusion method (inhibition zone of $17.33 \mathrm{~mm}$ ). With the broth microdilution method, $\mathrm{MIC}=3.12 \mu \mathrm{L} / \mathrm{mL}$ was found against $S$. aureus and all strains of S. epidermidis. C. aurantium was found to inhibit B. subtilis and P. crustosum [55]. A study on the antimicrobial activity of the $C$. aurantium EO against pathogenic bacteria (Staphylococcus aureus, Salmonella sp., Pseudomonas aeruginosa, Bacillus subtilis, and Escherichia coli) revealed that gram-positive bacteria were more susceptible than gram-negative bacteria [56].

G. procumbens L. EO exhibited the strongest antimicrobial activity against one strain of $S$. aureus with the disc diffusion test $(7.33 \mathrm{~mm})$. An MIC value of $12.50 \mu \mathrm{L} / \mathrm{mL}$ was found for S. aureus, S. capitis, and one strain of S. haemoliticus, determined with the broth microdilution method. Hammer et al. [57] reported a higher activity of G. procumbens EO against reference strains of gram-negative bacteria (Acinetobacter baumanii, Aeromonas sobria, Escherichia coli, Klebsiella pneumoniae, Salmonella typhi, and Serratia marcescens) observed in comparison to gram-positive microorganisms (Staphylococcus aureus and Enterococcus faecalis). A higher resistance of gram-positive bacteria against G. procumbens EO was shown by Nikolic et al. [24], who studied the bacteriostatic and bactericidal activity of the oil against microbial isolates.

L. cubeba (Lour.) Pers. EO exhibited the best antimicrobial activity against $S$. capitis with the disc diffusion test $(25.33 \mathrm{~mm})$ and an MIC of $0.39 \mu \mathrm{L} / \mathrm{mL}$ against $S$. aureus and S. capitis with the broth microdilitution test. The antibacterial activity of L. cubeba EO against food-borne pathogens has been reported as well [58-60]. A notably high antimicrobial activity was found against methicyllin-resistant Staphylococcus aureus (MRSA) [61,62].

The EOs of M. ericifolia Smith showed the strongest antimicrobial activity against $S$. aureus with respect to S. hominis. Melaleuca EOs have been reported to possess antibacterial activity against common food-borne pathogens [63] and were suggested for the eradication of MRSA in hospitals [64]. Even a concentration of $5 \%$ M. alternifolia was active against pathogenic bacteria of skin, and a potential application of M. alternifolia oil for wound treatment was suggested as well [65-67]. Furthermore, antimicrobial, antifungal, antiviral, and antioxidant properties were described in M. ericifolia [26]. Leaf extracts acquired from this plant exhibited antimicrobial activity against gram-positive and gram-negative bacteria, including S. aureus [68].

The EO of Pogestemon cabli was the most effective against two strains of S. haemoliticus and S. homins (inhibition zone of $12.67 \mathrm{~mm}$ ) using the disc diffusion method. The recorded MIC values against two strains of $S$. aureus, S. capitis, all strains of S. haemoliticus, and all but two strains of S. hominis were $3.12 \mu \mathrm{L} / \mathrm{mL}$. The EO from P. cabli was found to be more active against gram-positive than gram-negative bacteria, with the largest inhibition zone ( $35 \mathrm{~mm}$ with $20 \mu \mathrm{L}$ of oil) and the lowest MIC $(250 \mu \mathrm{g} / \mathrm{mL})$ and minimum bactericidal concentration (MBC) $(750 \mu \mathrm{g} / \mathrm{mL})$ found against Bacillus cereus. A moderate antifungal activity was recorded against Candida albicans in comparison to Saccharomyces 
cerevisiae (16- vs. 14-mm zone diameters with $20 \mu \mathrm{L}$ of oil). The lowest MIC and minimal fungicidal concentration(MFC) (both were $750 \mu \mathrm{g} / \mathrm{mL}$ ) were found for Candida albicans [69].

The EO of C. limon (L.) Osbeck was found to be the most effective against one strain of S. capitis, with an inhibition zone of $12.67 \mathrm{~mm}$ with the disc diffusion test. The broth microdilution method showed MICs of $3.12 \mu \mathrm{L} / \mathrm{mL}$ against S. aureus, S. capitis, as well as several strains of S. haemoliticus and S. hominis. The antimicrobial activity of EOs from C. limon was recorded against S. aureus, E. coli, and B. subtilis [70], with inhibitory effects against gram-positive bacteria [71]. Hydro-distillated EOs from C. limon were reported to be more active due to a high content of limonene [72].

The EOs of S. album L. exhibited the highest antimicrobial activity against one strain of S. hominis $(8.67 \mathrm{~mm})$. An MIC of $6.25 \mu \mathrm{L} / \mathrm{mL}$ was detected against S. capitis and all strains of S. hominis. A previously reported MIC for S. album ranged between 0.078 and $5 \mu \mathrm{g} / \mathrm{mL}$ [73], and an antimicrobial activity against Staphylococcus aureus and Klebsiella pneumoniae was described as well [74].

The EO of V. zizanioides (L.) Roberty showed the highest activity against $S$. capitis and one strain of $S$. homins with an inhibition zone of $12.67 \mathrm{~mm}$ using the disc diffusion test. With the broth microdilution tests, the MIC was $3.12 \mu \mathrm{L} / \mathrm{mL}$ against S. aureus, S. capitis, and all strains S. hominis. Gupta et al. [75] found a higher antimicrobial activity of the EO against gram-positive in comparison to gram-negative bacteria. Antifungal and antimicrobial activity against Candida albicans as well as wildtype and drug-resistant strains of $M$. smegmatis and drug-resistant strains of $E$. coli have been previously reported [76].

\section{Materials and Methods}

\subsection{Essential Oil Samples}

The following essential oils were used in the present study (Table 8): Amyris balsamifera L., Boswellia carterii Birdw., Canarium luzonicum (Blume) A. Gray, Cinnamomum camphora (L.) J. Presl., Cinnamomum camphora var. linaloolifera Y. Fuita, Citrus x aurantium L., Gaultheria procumbens L., Litsea cubeba (Lour.) Pers., Melaleuca ericifolia Smith., Melaleuca leucadendra L., Pogostemon cablin (Blanco) Benth., Citrus limon (L.) Osbeck, Santalum album L., and Vetiveria zizanoides (L.) Roberty. All EOs were produced in Slovakia (Hanus a.s., Nitra) and used in original packaging. All tested oils were stored in the dark at $4{ }^{\circ} \mathrm{C}$.

Table 8. List of essential oils.

\begin{tabular}{lccc}
\hline \multicolumn{1}{c}{ Botanical Species } & Common Name & Family & Part \\
\hline Amyris balsamifera L. & amyris & Rutaceae & crushed wood \\
Boswellia carterii Birdw. & frankincense & Burseraceae & resin \\
Canarium luzonicum (Blume) A. Gray & elemi & Burseraceae & resin \\
Cinnamomum camphora (L.) J. Presl. & camphor three bark & Lauraceae & wood, branches \\
Cinnamomum camphora var. linaloolifera Y. Fuita & ho leaf & Lauraceae & leaves \\
Citrus x aurantium L. & petitgrain & Rutaceae & leaves \\
Gaultheria procumbens L. & wintergreen & Ericaceae & leaves \\
Litsea cubeba (Lour.) Pers. & litsea cubeba fruit & Lauraceae & fruits \\
Melaleuca leucadendron L. & cajeput & Myrtaceae & shoots of leaves \\
Melaleuca ericifolia Smith. & rosalina & Myrtaceae & branches \\
Pogostemon cabli (Blanco) Benth. & patchouli & Lamiaceae & fermented leaves \\
Citrus limon (L.) Osbeck & lemon & Rutaceae & fruits \\
Santalum album L. & sandalwood & Santalaceae & crushed wood \\
Vetiveria zizanoides (L.) Roberty & vetiver & Poaceae & dried roots \\
\hline
\end{tabular}

\subsection{Chemical Composition of EOs}

Gas chromatographic-mass spectrometric analysis (GC Agilent 7890B and MS Agilent 5977A, Agilent Technologies Inc., Santa Clara, CA, USA) of the EOs was performed as described by Kačániová et al. [77] with a slightly modified version. Prior to the analysis, EO samples were diluted in hexane (HPLC $\geq 97 \%$, Sigma Aldrich GmbH, Darmstad, Germany) to a concentration of $10 \mu \mathrm{L} / \mathrm{mL}$. One microliter of diluted sample was injected into the inlet $\left(250^{\circ} \mathrm{C}\right)$ operated in split mode 
1:10. The separation was achieved using a HP-5ms capillary column $(30 \mathrm{~m} \times 0.25 \mathrm{~mm} \times 0.25 \mu \mathrm{m}$ film; Agilent Technologies). The oven temperature program was set to $50{ }^{\circ} \mathrm{C}$ for the first $5 \mathrm{~min}$ and subsequently increased to $240{ }^{\circ} \mathrm{C}$ at the rate of $3{ }^{\circ} \mathrm{C} / \mathrm{min}$, where it was kept constant for $2 \mathrm{~min}$. Helium was used as a carrier gas at constant flow $(1.2 \mathrm{~mL} / \mathrm{min})$. The mass detector parameters were as follows: ionization energy of the filament $-70 \mathrm{eV}$, transfer line temperature $-250{ }^{\circ} \mathrm{C}$, MS source temperature $-230^{\circ} \mathrm{C}$, and quadrupole temperature- $150^{\circ} \mathrm{C}$. The mass spectrometer was programmed under electron impact (EI) in a full scan mode at $m / z$ 40-350 with a scanning rate of $2.4 \mathrm{scans} / \mathrm{s}$. The identification of compounds was carried out by comparing mass spectra (over $80 \%$ match) with a commercial database NIST ${ }^{\circledR} 2017$ and the Wiley library for retention times of reference standards (D-limonene, $\beta$-myrcene, and $\gamma$-terpinene; Sigma-Aldrich $\mathrm{GmbH}$ ) to compare data on occurrence in EOs with the literature. The relative content of the identified compounds was calculated by dividing the individual peak area by the total area of all peaks. Peaks under $1 \%$ were not counted. Each sample was measured in triplicate.

\subsection{Microorganisms}

Semen samples were obtained from 27 males following 2 days of sexual abstinence. The specimens were taken by masturbation into a sterile wide mouth container. The samples were liquefied at $37^{\circ} \mathrm{C}$ for $30 \mathrm{~min}$. All experiments were performed within $1 \mathrm{~h}$ after sampling. Only ejaculates showing normal semen parameters (concentration $>20 \times 10^{6} / \mathrm{mL}$, motility $>40 \%$, viability $>40 \%$, and morphology $>4 \%$ ) and free from leukocytes were used. The experiments were approved by the Ethic Committee at the Specialized Hospital Sv. Svodar Zobor, protocol no. 030809/2015. Tryptone Soya agar (TSA, Merck, Darmstadt, Germany) and Blood agar (BA, Merck, Darmstadt, Germany) were inoculated with the semen samples, and after incubation $\left(24 \mathrm{~h}, 37^{\circ} \mathrm{C}\right)$, individual colonies were selected for further confirmation with MALDI-TOF MS Biotyper (Brucker Daltonics, Bremen, Germany) [78]. The isolates were maintained in Mueller Hinton Agar (MHA, Merck, Darmstadt, Germany) and cultured $24 \mathrm{~h}$ before the experiment to reach a concentration of $10^{5} \mathrm{cfu} / \mathrm{mL}$.

\subsection{Antimicrobial Susceptibility Testing}

The antimicrobial susceptibility test was performed with the disc diffusion method against $(10 \mathrm{mcg})$ chloramphenicol, tetracycline, tigecycline, and tobramycin. The discs were obtained from Oxoid (Basingstoke, UK). The results were interpreted according to EUCAST [36].

\subsection{Disc Diffusion Method}

A suspension of the tested culture $\left(0.1 \mathrm{~mL}\right.$ of $10^{5}$ cells $\left./ \mathrm{mL}\right)$ was spread onto Mueller Hinton Agar (MHA, Oxoid, Basingstoke, UK). Filter paper discs $(6 \mathrm{~mm})$ were impregnated with $15 \mu \mathrm{L}$ of the EO and placed on the inoculated plates. The agars were incubated at $4{ }^{\circ} \mathrm{C}$ for $2 \mathrm{~h}$ and subsequently placed into an incubator at $37^{\circ} \mathrm{C}$ for $24 \mathrm{~h}$. The diameters of the inhibition zones were measured in $\mathrm{mm}$. All the tests were performed in triplicate [79]. The results were evaluated as follows (disk diameter included): $\geq 15 \mathrm{~mm}$ was strongly inhibitory, $<15-10 \mathrm{~mm}$ was moderately/mildly inhibitory, and $<10 \mathrm{~mm}$ was not inhibitory [78-82].

\subsection{Determination of Minimum Inhibitory Concentration}

The broth microdilution assay was used for determination of the minimal inhibition concentration (MIC) according to the Clinical and Laboratory Standards Institute [83]. All tests were performed in Mueller Hinton Broth (MHB, Oxoid, Basingstoke, UK). The bacterial strains were cultured overnight at $37^{\circ} \mathrm{C}$ in MHA. The tested strains were suspended in MHB to give a final density of $10^{6} \mathrm{cfu} / \mathrm{mL}$ confirmed by viable counts. The EO solution was prepared in dimethyl sulphoxide (DMSO, Penta, Prague, Czech Republic). An amount of $50 \mu \mathrm{L}$ of MHB was added to each 96-well micro-titer plate, and $100 \mu \mathrm{L}$ of MHB was added to the 10th well for sterility control. For the growth control, MHB with $5 \%$ DMSO was added to the 9th well. Fifty microliters of EOs initially dissolved in 5\% DMSO 
were added into the first well. A serial 2-fold dilution was performed by transferring $50 \mu \mathrm{L}$ of the suspension to the subsequent wells up to the 8 th well; bacterial inoculum of $0.5 \mathrm{McFarland}$ was diluted in the ratio of 1:100 and added into the 1st-8th wells in order to acheive the final concentration of $5 \times 10^{5} \mathrm{cfu} / \mathrm{mL}$. Bacterial cell viability and MIC values were determined by observing the turbidity. The lowest concentrations of the EOs with clear suspension were considered as the MIC values. The test was performed in triplicate alongside cefoxitin $(30 \mathrm{mcg})$, used as a positive control.

\subsection{Statistical Analysis}

The basic variation (disc diffusion method) in statistical values from obtained data were calculated with Statgraphic, Tukey HSD test. Mean, standard deviation, minimum, maximum, coefficient of variation, and frequency of size of inhibition zones were calculated for the antimicrobial activity of essential oils.

\section{Conclusions}

In this study, 50 different strains of Staphylococcus spp. isolated from human semen were tested for susceptibility against 14 different essential oils alongside determination of their chemical composition. The antimicrobial resistance of the tested isolates was evaluated as well. The antimicrobial resistance of Staphylococcus spp. against chloramphenicol and tobramycin was found, while all isolates were sensitive to tetracycline and tigecycline. C. luzonicum (Blume) A. Gray exhibited a strong inhibitory effect; A. balsamifera L., C. camphora var. linaloolifera Y. Fuita, L. cubeba (Lour.) Pers., and P. cabli (Blanco) Benth. possessed a moderately inhibitory effect; and B. carterii Birdw., C. camphora (L.) J. Presl., C. aurantium L., G. procumbens L., M. leucadendron L., M. ericifolia Smith., C. limon (L.) Osbeck, S. album L., and V. zizanoides (L.) Roberty revealed no inhibitory activity on Staphylococcus spp. isolated from human ejaculates. As such, we may suggest the use of the selected essential oils against Staphylococcus spp. contamination of human semen samples.

Author Contributions: Conceptualization, M.K. and E.T.; data curation, M.K., M.T., J.Ž., J.Š., P.Ł.K., and N.V.; investigation, M.K., M.T., J.Ž., T.S., and D.G.; methodology, M.K.; supervision, M.K.; writing—original draft, M.K., M.T., E.T., J.Š., J.Ž., T.S., D.G., P.Ł.K., and N.V. All authors have carefully revised and approved the final version of the manuscript.

Funding: This work was supported by grants APVV SK-BY-RD-19-0014 and BRFBR № X20SLKG-003 "The formulation of novel compositions and properties study of the polysaccharide-based edible films and coatings with antimicrobial and antioxidant plant additives".

Acknowledgments: The paper was supported by the Slovak Research and Development Agency project no. APVV-15-0544.

Conflicts of Interest: The authors declare no conflict of interest.

\section{References}

1. Pellati, D.; Mylonakis, I.; Bertoloni, G.; Fiore, C.; Andrisani, A.; Ambrosini, G.; Armanini, D. Genital tract infections and infertility. Eur. J. Obstet. Gynecol. Reprod. Biol. 2008, 140, 3-11. [CrossRef]

2. Henkel, R.; Schill, W.-B. Sperm separation in patients with urogenital infections. Andrologia 2009, 30, 91-97. [CrossRef] [PubMed]

3. Urata, K.; Narahara, H.; Tanaka, Y.; Egashira, T.; Takayama, F.; Miyakawa, I. Effect of endotoxin-induced reactive oxygen species on sperm motility. Fertil. Steril. 2001, 76, 163-166. [CrossRef]

4. Sanocka-Maciejewska, D.; Ciupińska, M.; Kurpisz, M. Bacterial infection and semen quality. J. Reprod. Immunol. 2005, 67, 51-56. [CrossRef] [PubMed]

5. Purvis, K.; Christiansen, E. Infection in the male reproductive tract. Impact, diagnosis and treatment in relation to male infertility. Int. J. Androl. 1993, 16, 1-13. [CrossRef]

6. Marconi, M.; Pilatz, A.; Wagenlehner, F.; Diemer, T.; Weidner, W. Impact of infection on the secretory capacity of the male accessory glands. Int. Braz. J. Urol. 2009, 35, 299-309. [CrossRef] [PubMed] 
7. Greive, K.A.; Barnes, T.M. The efficacy of Australian essential oils for the treatment of head lice infestation in children: A randomised controlled trial. Australas. J. Dermatol. 2018, 59, e99-e105. [CrossRef]

8. Brochot, A.; Guilbot, A.; Haddioui, L.; Roques, C. Antibacterial, antifungal, and antiviral effects of three essential oil blends. Microbiologyopen 2017, 6, e00459. [CrossRef]

9. Man, A.; Gâz, A.Ş.; Mare, A.D.; Berţa, L. Effects of low-molecular weight alcohols on bacterial viability. Rev. Rom. Med. Lab. 2017, 25, 335-343. [CrossRef]

10. Kačániová, M.; Ivanišová, E.; Ondrejovič, M.; Chmelová, D.; Terentjeva, M.; Kunová, S.; Tvrdá, E. BIological activity of essential oils against Staphylococcus spp. isolated from human semen. J. Microbiol. Biotechnol. Food Sci. 2019, 9, 404-408. [CrossRef]

11. Salari, M.H.; Amine, G.; Shirazi, M.H.; Hafezi, R.; Mohammadypour, M. Antibacterial effects of Eucalyptus globulus leaf extract on pathogenic bacteria isolated from specimens of patients with respiratory tract disorders. Clin. Microbiol. Inf. 2006, 12, 194-196. [CrossRef] [PubMed]

12. Fraczek, M.; Kurpisz, M. Mechanisms of the harmful effects of bacterial semen infection on ejaculated human spermatozoa: Potential inflammatory markers in semen. Folia Histochem. Cytobiol. 2015, 53, 201-217. [CrossRef] [PubMed]

13. Wallach, E.E.; Wolff, H. The biologic significance of white blood cells in semen. Fertil. Steril. 1995, 63, 1143-1157. [CrossRef]

14. Khalili, M.; Sharifi-Yazdi, M. The effect of bacterial infection on the quality of human's spermatozoa. Iran. J. Public Health 2001, 35, 62-67.

15. Barra, A. Factors affecting chemical variability of essential oils: A review of recent developments. Nat. Prod. Commun. 2009, 4. [CrossRef]

16. Kloucek, P.; Smid, J.; Flesar, J.; Havlik, J.; Titera, D.; Rada, V.; Drabek, O.; Kokoska, L. In vitro inhibitory activity of essential oil vapors against Ascosphaera apis. Nat. Prod. Commun. 2012, 7. [CrossRef]

17. Camarda, L.; Dayton, T.; Di Stefano, V.; Pitonzo, R.; Schillaci, D. Chemical composition and antimicrobial activity of some oleogum resin essential oils from Boswellia spp. (Burseraceae). Ann. Chim. 2007, 97, 837-844. [CrossRef]

18. Villanueva, M.A.; Torres, R.C.; Başer, K.H.C.; Özek, T.; Kürkçüoğlu, M. The composition of Manila elemi oil. Flavour Fragr. J. 1993, 8, 35-37. [CrossRef]

19. Liang, G.Y.; Qiu, D.W.; Wei, H.F.; Li, H.Y.; Zhao, S.; He, Z.Y.; Liu, N. The essential oil of the fruits from Cinnamomum camphora. J. Guiyang Coll. Tradit. Chin. Med. 1994, 16, 59-60.

20. Li, S.L.; Luo, Y. The analysis of essential oil from camphor tree fruits in Jinggangshan. J. Jiangxi Univ. Tradit. Chin. Med. 2005, 17, 36-37.

21. Frizzo, C.D.; Santos, A.C.; Paroul, N.; Serafini, L.A.; Dellacassa, E.; Lorenzo, D.; Moyna, P. Essential oils of camphor tree (Cinnamomum camphora nees \& eberm) cultivated in Southern Brazil. Braz. Arch. Biol. Technol. 2000, 43, 313-316. [CrossRef]

22. Macleod, A.J.; Macleod, G.; Subramanian, G. Volatile aroma constituents of orange. Phytochemistry 1988, 27, 2185-2188. [CrossRef]

23. Kabouche, Z.; Benkiki, N.; Seguin, E.; Bruneau, C. A new dicoumarinyl ether and two rare furocoumarins from Ruta montana. Fitoterapia 2003, 74, 194-196. [CrossRef]

24. Nikolić, M.; Marković, T.; Mojović, M.; Pejin, B.; Savić, A.; Perić, T.; Marković, D.; Stević, T.; Soković, M. Chemical composition and biological activity of Gaultheria procumbens L. essential oil. Ind. Crops Prod. 2013, 49,561-567. [CrossRef]

25. Thielmann, J.; Muranyi, P. Review on the chemical composition of Litsea cubeba essential oils and the bioactivity of its major constituents citral and limonene. J. Essent. Oil Res. 2019, 31, 361-378. [CrossRef]

26. Pujiarti, R.; Ohtani, Y.; Ichiura, H. Physicochemical properties and chemical compositions of Melaleuca leucadendron leaf oils taken from the plantations in Java, Indonesia. J. Wood Sci. 2011, 57, 446-451. [CrossRef]

27. An, N.T.G.; Huong, L.T.; Satyal, P.; Tai, T.A.; Dai, D.N.; Hung, N.H.; Ngoc, N.T.B.; Setzer, W.N. Mosquito larvicidal activity, antimicrobial activity, and chemical compositions of essential oils from four species of myrtaceae from Central Vietnam. Plants 2020, 9, 544. [CrossRef]

28. Silva, C.J.; Barbosa, L.C.A.; Maltha, C.R.A.; Pinheiro, A.L.; Ismail, F.M.D. Comparative study of the essential oils of seven Melaleuca (Myrtaceae) species grown in Brazil. Flavour Fragr. J. 2007, 22, 474-478. [CrossRef]

29. Farag, R.S.; Shalaby, A.S.; El-Baroty, G.A.; Ibrahim, N.A.; Ali, M.A.; Hassan, E.M. Chemical and biological evaluation of the essential oils of different Melaleuca species. Phyther. Res. 2004, 18, 30-35. [CrossRef] 
30. Sakasegawa, M.; Hori, K.; Yatagai, M. Composition and antitermite activities of essential oils from Melaleuca species. J. Wood Sci. 2003, 49, 181-187. [CrossRef]

31. Murugan, R.; Mallavarapu, G.R.; Padmashree, K.V.; Rao, R.R.; Livingstone, C. Volatile oil composition of Pogostemon heyneanus and comparison of its composition with patchouli oil. Nat. Prod. Commun. 2010, 5. [CrossRef]

32. Paw, M.; Begum, T.; Gogoi, R.; Pandey, S.K.; Lal, M. Chemical composition of Citrus limon L. burmf peel essential oil from North East India. J. Essent. Oil Bear. Plants 2020, 23, 337-344. [CrossRef]

33. Subasinghe, U.; Gamage, M.; Hettiarachchi, D.S. Essential oil content and composition of Indian sandalwood (Santalum album) in Sri Lanka. J. For. Res. 2013, 24, 127-130. [CrossRef]

34. Teixeira da Silva, J.A.; Kher, M.M.; Soner, D.; Page, T.; Zhang, X.; Nataraj, M.; Ma, G. Sandalwood: Basic biology, tissue culture, and genetic transformation. Planta 2016, 243, 847-887. [CrossRef]

35. David, A.; Wang, F.; Sun, X.; Li, H.; Lin, J.; Li, P.; Deng, G. Chemical composition, antioxidant, and antimicrobial activities of Vetiveria zizanioides (L.) nash essential oil extracted by carbon dioxide expanded ethanol. Molecules 2019, 24, 1897. [CrossRef]

36. EUCAST. The European Committee on Antimicrobial Susceptibility Testing. Available online: http:// www.eucast.org/fileadmin/src/media/PDFs/EUCAST_files/Breakpoint_tables/v_9.0_Breakpoint_Tables.pdf (accessed on 13 September 2020).

37. Schwarz, S.; Kehrenberg, C.; Doublet, B.; Cloeckaert, A. Molecular basis of bacterial resistance to chloramphenicol and florfenicol. FEMS Microbiol. Rev. 2004, 28, 519-542. [CrossRef]

38. Schwarz, S.; White, D.G. Phenicol resistance. In Frontiers in Antimicrobial Resistance: A Tribute to Stuart B. Levy; White, D.G., Alekshun, M.N., McDermott, P.F., Eds.; ASM Press: Washington, DC, USA, 2005; pp. 124-147.

39. Witte, W. Biomedicine: Medical consequences of antibiotic use in agriculture. Science 1998, 279, 996-997. [CrossRef]

40. Falkiner, F. The consequences of antibiotic use in horticulture. J. Antimicrob. Chemother. 1998, 41, 429-431. [CrossRef]

41. Levy, S.B. The Antibiotic Paradox; Springer: Boston, MA, USA, 1992; ISBN 978-0-306-44331-2.

42. Roberts, M.C. Tetracycline resistance determinants: Mechanisms of action, regulation of expression, genetic mobility, and distribution. FEMS Microbiol. Rev. 1996, 19, 1-24. [CrossRef]

43. Biedenbach, D.J.; Beach, M.L.; Jones, R.N. In vitro antimicrobial activity of GAR-936 tested against antibiotic-resistant gram-positive blood stream infection isolates and strains producing extended-spectrum $\beta$-lactamases. Diagn. Microbiol. Infect. Dis. 2001, 40, 173-177. [CrossRef]

44. Edlund, C.; Nord, C.E. In-vitro susceptibility of anaerobic bacteria to gar-936, a new glycylcycline. Clin. Microbiol. Infect. 2000, 6, 159-163. [CrossRef] [PubMed]

45. Sanfilippo, C.M.; Morrissey, I.; Janes, R.; Morris, T.W. Surveillance of the activity of aminoglycosides and fluoroquinolones against ophthalmic pathogens from Europe in 2010-2011. Curr. Eye Res. 2015, 1-9. [CrossRef] [PubMed]

46. Dahiya, P.; Manglik, A. Evaluation of antibacterial, antifungal and antioxidant potential of essential oil from Amyris balsamifera against multi drug resistant clinical isolates. Asian J. Pharm. Clin. Res. 2013, 6, 57-60.

47. Van Vuuren, S.F.; Kamatou, G.P.P.; Viljoen, A.M. Volatile composition and antimicrobial activity of twenty commercial frankincense essential oil samples. S. Afr. J. Bot. 2010, 76, 686-691. [CrossRef]

48. Screening of the antifungal activity of essential oils against human and plant pathogenic filamentous fungi. Flora Mediterr. 2019, 29. [CrossRef]

49. Satyal, P.; Paudel, P.; Poudel, A.; Dosoky, N.S.; Pokharel, K.K.; Setzer, W.N. Bioactivities and compositional analyses of Cinnamomum essential oils from Nepal: C. camphora, C. tamala, and C. glaucescens. Nat. Prod. Commun. 2013, 8, 1777-1784. [CrossRef]

50. Mishra, A.K.; Dwivedi, S.K.; Kishore, N.; Dubey, N.K. Fungistatic properties of essential oil of Cinnamomum camphora. Int. J. Pharmacogn. 1991, 29, 259-262. [CrossRef]

51. Liu, C.H.; Mishra, A.K.; He, B.; Tan, R.X. Composition and antifungal activity of essential oils from Artemisia princeps and Cinnamomum camphora. Int. Pest Control 2001, 43, 72-74.

52. Singh, P.; Srivastava, B.; Kumar, A.; Dubey, N.K. Fungal contamination of raw materials of some herbal drugs and recommendation of Cinnamomum camphora oil as herbal fungitoxicant. Microb. Ecol. 2008, 56, 555-560. [CrossRef] 
53. Ho, C.-L.; Eugene, I.; Wang, C.; Su, Y.-C. Essential oil compositions and bioactivities of the various parts of Cinnamomum camphora Sieb. var. linaloolifera Fujuta. For. Res. Q. 2009, 31, 77-95.

54. Rangari, P. Screening of antibacterial sensitivty of essential oils of camphor and cinnamon. Shodh Anusandhan Samachar 2011, 1, 22-26.

55. Kačániová, M.; Terentjeva, M.; Galovičová, L.; Ivanišová, E.; Štefániková, J.; Valková, V.; Borotová, P.; Kowalczewski, P.Ł.; Kunová, S.; Felšöciová, S.; et al. Biological activity and antibiofilm molecular profile of Citrus aurantium essential oil and its application in a food model. Molecules 2020, 25, 3956. [CrossRef] [PubMed]

56. Teneva, D.; Denkova-Kostova, R.; Goranov, B.; Hristova-Ivanova, Y.; Slavchev, A.; Denkova, Z.; Kostov, G. Chemical composition, antioxidant activity and antimicrobial activity of essential oil from Citrus aurantium $\mathrm{L}$. zest against some pathogenic microorganisms. Z. Nat. C 2019, 74, 105-111. [CrossRef]

57. Hammer, K.A.; Carson, C.F.; Riley, T.V. Antimicrobial activity of essential oils and other plant extracts. J. Appl. Microbiol. 1999, 86, 985-990. [CrossRef] [PubMed]

58. Li, W.-R.; Shi, Q.-S.; Liang, Q.; Xie, X.-B.; Huang, X.-M.; Chen, Y.-B. Antibacterial activity and kinetics of Litsea cubeba oil on Escherichia coli. PLoS ONE 2014, 9, e110983. [CrossRef]

59. Liu, T.-T.; Yang, T.-S. Antimicrobial impact of the components of essential oil of Litsea cubeba from Taiwan and antimicrobial activity of the oil in food systems. Int. J. Food Microbiol. 2012, 156, 68-75. [CrossRef] [PubMed]

60. Yang, Y.; Jiang, J.; Qimei, L.; Yan, X.; Zhao, J.; Yuan, H.; Qin, Z.; Wang, M. The fungicidal terpenoids and essential oil from Litsea cubeba in Tibet. Molecules 2010, 15, 7075-7082. [CrossRef]

61. Cui, H.; Li, W.; Li, C.; Vittayapadung, S.; Lin, L. Liposome containing cinnamon oil with antibacterial activity against methicillin-resistant Staphylococcus aureus biofilm. Biofouling 2016, 32, 215-225. [CrossRef]

62. Hu, W.; Li, C.; Dai, J.; Cui, H.; Lin, L. Antibacterial activity and mechanism of Litsea cubeba essential oil against methicillin-resistant Staphylococcus aureus (MRSA). Ind. Crops Prod. 2019, 130, 34-41. [CrossRef]

63. Siddique, S.; Parveen, Z.; Firdaus-e-Bareen; Mazhar, S. Chemical composition, antibacterial and antioxidant activities of essential oils from leaves of three Melaleuca species of Pakistani flora. Arab. J. Chem. 2020, 13, 67-74. [CrossRef]

64. Dryden, M.; Dailly, S.; Crouch, M. A randomized, controlled trial of tea tree topical preparations versus a standard topical regimen for the clearance of MRSA colonization. J. Hosp. Infect. 2004, 56, 283-286. [CrossRef] [PubMed]

65. Messager, S.; Hammer, K.A.; Carson, C.F.; Riley, T.V. Effectiveness of hand-cleansing formulations containing tea tree oil assessed ex vivo on human skin and in vivo with volunteers using European standard EN 1499. J. Hosp. Infect. 2005, 59, 220-228. [CrossRef]

66. Edmondson, M.; Newall, N.; Carville, K.; Smith, J.; Riley, T.V.; Carson, C.F. Uncontrolled, open-label, pilot study of tea tree (Melaleuca alternifolia) oil solution in the decolonisation of methicillin-resistant Staphylococcus aureus positive wounds and its influence on wound healing. Int. Wound J. 2011, 8, 375-384. [CrossRef] [PubMed]

67. Blackwood, B.; Thompson, G.; McMullan, R.; Stevenson, M.; Riley, T.V.; Alderdice, F.A.; Trinder, T.J.; Lavery, G.G.; McAuley, D.F. Tea tree oil (5\%) body wash versus standard care (Johnson's Baby Softwash) to prevent colonization with methicillin-resistant Staphylococcus aureus in critically ill adults: A randomized controlled trial. J. Antimicrob. Chemother. 2013, 68, 1193-1199. [CrossRef]

68. Hussein, S.A.M.; Hashim, A.N.M.; El-Sharawy, R.T.; Seliem, M.A.; Linscheid, M.; Lindequist, U.; Nawwar, M.A.M. Ericifolin: An eugenol 5-O-galloylglucoside and other phenolics from Melaleuca ericifolia. Phytochemistry 2007, 68, 1464-1470. [CrossRef]

69. Das, P.; Dutta, S.; Begum, J.; Anwar, M.N. Antibacterial and antifungal activity analysis of essential oil of Pogostemon cablin (Blanco) benth. Bangladesh J. Microbiol. 2016, 30, 7-10. [CrossRef]

70. Saeb, S.; Amin, M.; Seyfi Gooybari, R.; Aghel, N. Evaluation of antibacterial activities of Citrus limon, Citrus reticulata, and Citrus grandis against pathogenic bacteria. Int. J. Enteric Pathog. 2016, 4, 11-15. [CrossRef]

71. Dhiman, A.; Ahmad, S.; Nanda, A.; Narasimhan, B. In vitro antimicrobial status of methanolic extract of Citrus sinensis Linn. fruit peel. Chron. Young Sci. 2012, 3, 204. [CrossRef]

72. Hamdan, D.; Ashour, M.L.; Mulyaningsih, S.; El-Shazly, A.; Wink, M. Chemical composition of the essential oils of variegated pink-fleshed lemon (Citrus $\times$ limon L. Burm. f.) and their anti-inflammatory and antimicrobial activities. Z. Nat. C 2013, 68, 0275. [CrossRef] 
73. Misra, B.B.; Dey, S. Comparative phytochemical analysis and antibacterial efficacy of in vitro and in vivo extracts from East Indian sandalwood tree (Santalum album L.). Lett. Appl. Microbiol. 2012, 55, 476-486. [CrossRef]

74. Jirovetz, L.; Buchbauer, G.; Denkova, Z.; Stoyanova, A.; Murgov, I.; Gearon, V.; Birkbeck, S.; Schmidt, E.; Geissler, M. Comparative study on the antimicrobial activities of different sandalwood essential oils of various origin. Flavour Fragr. J. 2006, 21, 465-468. [CrossRef]

75. Gupta, R.; Sharma, K.K.; Afzal, M.; Damanhouri, Z.A.; Ali, B.; Kaur, R.; Kazmi, I.; Anwar, F. Anticonvulsant activity of ethanol extracts of Vetiveria zizanioides roots in experimental mice. Pharm. Biol. 2013, 51, 1521-1524. [CrossRef] [PubMed]

76. Champagnat, P.; Sidibé, L.; Forestier, C.; Carnat, A.; Chalchat, J.-C.; Lamaison, J.-L. Antimicrobial activity of essential oils from Vetiveria nigritana and Vetiveria zizanioides roots. J. Essent. Oil Bear. Plants 2007, 10, 519-524. [CrossRef]

77. Kačániová, M.; Galovičová, L.; Ivanišová, E.; Vukovic, N.L.; Štefániková, J.; Valková, V.; Borotová, P.; Žiarovská, J.; Terentjeva, M.; Felšöciová, S.; et al. Antioxidant, antimicrobial and antibiofilm activity of coriander (Coriandrum sativum L.) essential oil for its application in foods. Foods 2020, 9, 282. [CrossRef] [PubMed]

78. Kačániová, M.; Kunová, S.; Sabo, J.; Ivanišová, E.; Žiarovská, J.; Felšöciová, S.; Fatrcová-Šramková, K.; Terentjeva, M. Isolation and identification of lactic acid bacteria in wine production by MALDI-TOF MS biotyper. Acta Hortic. Regiotect. 2020, 23, 21-24. [CrossRef]

79. Zheljazkov, V.D.; Sikora, V.; Semerdjieva, I.B.; Kačániová, M.; Astatkie, T.; Dincheva, I. Grinding and fractionation during distillation alter hemp essential oil profile and its antimicrobial activity. Molecules 2020, 25, 3943. [CrossRef]

80. Xiangwei, Z.; Xiaodong, W.; Peng, N.; Yang, Z.; JiaKuan, C. Chemical composition and antimicrobial activity of the essential oil of Sagittaria trifolia. Chem. Nat. Compd. 2006, 42, 520-522. [CrossRef]

81. Rota, M.C.; Herrera, A.; Martínez, R.M.; Sotomayor, J.A.; Jordán, M.J. Antimicrobial activity and chemical composition of Thymus vulgaris, Thymus zygis and Thymus hyemalis essential oils. Food Control 2008, 19, 681-687. [CrossRef]

82. Lv, F.; Liang, H.; Yuan, Q.; Li, C. In vitro antimicrobial effects and mechanism of action of selected plant essential oil combinations against four food-related microorganisms. Food Res. Int. 2011, 44, 3057-3064. [CrossRef]

83. National Committee for Clinical Laboratory Standards. Performance Standards for Antimicrobial Susceptibility Testing; 9th International Supplement, M100-S9; NCCLS: Wayne, PA, USA, 1999.

Publisher's Note: MDPI stays neutral with regard to jurisdictional claims in published maps and institutional affiliations.

(C) 2020 by the authors. Licensee MDPI, Basel, Switzerland. This article is an open access article distributed under the terms and conditions of the Creative Commons Attribution (CC BY) license (http://creativecommons.org/licenses/by/4.0/). 